\title{
Basis set truncation corrections for improved frozen natural orbital $\operatorname{CCSD}(\mathrm{T})$ energies
}

\author{
Péter R. Nagy, László Gyevi-Nagy, and Mihály Kállay \\ Department of Physical Chemistry and Materials Science, Budapest University of \\ Technology and Economics, H-1521 Budapest, P.O.Box 91, Hungary
}

\section{ARTICLE HISTORY}

Compiled July 26, 2021

\begin{abstract}
A number of approaches are proposed and assessed to reduce the frozen natural orbital (FNO) truncation error of coupled-cluster singles and doubles with perturbative triples $[\mathrm{CCSD}(\mathrm{T})]$ energies. The diagrammatic energy decomposition method of Irmler and Grüneis [J. Chem. Phys. 151, 104107 (2019)] is extended to the FNO truncation correction of the particle-particle ladder (PPL) term in the case of closedand open-shell molecular systems. The approach is tested for reaction and interaction energies, as well as atomization processes, and it is found the most robust for a wider range of FNO truncation thresholds outperforming the commonly employed additive MP2 correction. We also show that the linear extrapolation (LE) of FNO-CCSD(T) energies as a function of second-order Møller-Plesset (MP2) energies provides the best correlation energies and most balanced energy differences with tighter FNO thresholds, but it lacks systematic error compensation that would be required for the better performance with looser FNO thresholds. Further insight is gained from a diagrammatic and spin-component decomposition based analysis. Moreover, orbital (pair) specific energy decompositions are utilized to introduce size-consistent variants of the promising PPL and LE FNO corrections and their analogues for $(\mathrm{T})$, which are also readily applicable in the context of popular local correlation methods.
\end{abstract}

\section{KEYWORDS}

frozen natural orbitals, corrections for basis set truncation, coupled-cluster theory, $\operatorname{CCSD}(\mathrm{T})$

\section{Introduction}

If accurate and reliable results are desired in computational chemistry, high-level electron correlation methods should be deployed. In particular, the coupled-cluster (CC) hierarchy of methods $[1-3]$ has the potential to provide results with chemical accuracy $(\sim 1 \mathrm{kcal} / \mathrm{mol})$. However, these approaches are only affordable for relatively small systems because of the steep scaling of their computational expenses with the size of the system. Even the simple CC singles and doubles (CCSD) method [4] scale as $\mathcal{N}^{6}$, where $\mathcal{N}$ is a measure of the system size, while the expenses of the more advanced CCSD with perturbative triples [CCSD(T)] approach [5], which is usually required for the $1 \mathrm{kcal} / \mathrm{mol}$ accuracy goal, scale as $\mathcal{N}^{7}$. The situation is also aggravated by the 
slow basis set convergence of these methods. For a quantitative accuracy, rather large one-electron basis sets are needed. Considering that the rate-determining operations, for a given number of electrons, scale as the fourth power of the basis set size for both CCSD and the perturbative triples correction, the size of the basis also puts a limit on the applicability of the methods.

Over the years, several approaches have been developed to tackle these problems. Noteworthy are the CC implementations exploiting the density fitting (DF) approximation [6] or various parallelized CC algorithms [7-10], which do not change the scaling of the methods but decrease its prefactor. Nevertheless, the scaling can also be reduced to a lower power of $\mathcal{N}$, for example, by data compressing techniques [11] or even to linear using local correlation approximations [12-16]. The acceleration of the slow basis set convergence of the $\mathrm{CC}$ methods has also been the subject of numerous studies. It has been proven that the complete basis set (CBS) limit of conventional CC energies can be well approximated by basis set extrapolation techniques [17]. Finite basis CC energies can also be improved using basis set corrections obtained at a lower level of theory [18] or at different points of the potential energy surface. [19] An alternative solution to this problem is available at the CCSD level in the form of explicitly correlated approaches [20-22], which explicitly contain the interelectronic distances in the wave functions.

An alternative strategy to mitigate the basis set issue is offered by the various methods based on the transformation and subsequent truncation of the one-electron basis set. In these approaches, the virtual molecular orbital (MO) space is divided into an active and an inactive subspace, and the orbitals of the latter are neglected in the more demanding parts of the correlation calculation. To minimize the correlation energy loss, a transformation of the MOs is carried out in the virtual space before its truncation. In particular approaches, the extremum of a functional depending on the orbital rotation parameters of the virtual space is sought. The resulting modified virtual MOs are usually referred to as the optimized virtual orbitals (OVOs) [23-26]. Several functional forms have been proposed including modified second-order Hylleraas-functionals [23-25] and other ones that utilize the overlap of the CC wave functions expanded in the full and the active virtual spaces $[26,27]$. The benefits of using CCSD amplitudes instead of MP1 amplitudes to define the retained virtual subspace have also been explored[28].

A simpler but similarly efficient approach to compress the virtual space is the frozen natural orbital (FNO) approximation. In this scheme, the orbital transformation matrix is obtained by diagonalizing a one-particle density matrix constructed from a lower-level wave function. The resulting MOs are referred to as the natural orbitals (NOs), whereas the corresponding eigenvalues are interpreted as their populations [29-34]. The NO basis is usually favored over other bases because it generally gives the fastest convergence of the configuration interaction expansion [29]. In the FNO approach, the active space is assembled by neglecting the weakly populated NOs, that is, the orbitals with small eigenvalues. If we intend to reduce the costs of CCSD or $\operatorname{CCSD}(\mathrm{T})$, probably the best choice is to employ the NOs obtained with the firstorder Møller-Plesset (MP) wave function [25, 35-37]. Though the OVO and FNO approaches exhibited similar performance in benchmark studies [38, 39], thanks to its simplicity, the latter gained wider acceptance. It was extended to larger systems relying on reduced-scaling density matrix construction techniques [40-43], FNO-CC analytic gradients were implemented [36], and various excited-state extensions are also available [37, 44-47]. Besides the cost-reduction of conventional CC methods, various types of NOs are widely used to speed up local CC approaches. These include the pair 
natural orbitals (PNOs) [13-15, 28] and local natural orbitals (LNOs) [48], which are pair- and orbital-specific NOs, respectively, evaluated from density matrix fragments.

The error of the FNO approach introduced by the basis set compression can be significantly reduced via simple corrections. The most straightforward approach utilizes the second-order MP (MP2) energies calculated with the full virtual space and with the FNO basis. Both of these quantities are readily available, and allow the estimation of the truncation error at the MP2 level $[23,35]$. This scheme will be denoted by $\triangle \mathrm{MP} 2$. It was demonstrated in several studies that this correction remarkably improves the performance of the FNO method due to error compensation [36, 49]. The $\triangle \mathrm{MP} 2$ correction also turned out to be very efficient in our LNO-CC methods $[12,48]$ to reduce the LNO truncation error in the orbital-specific domains of localized MOs. The FNO approximation can also be corrected by extrapolation of the correlation energy. Several techniques have been proposed along this line $[37,50]$ including also our recent study [51]. In the PNO framework, the asymptotic convergence of PNO expansions was studied by Petersson and co-workers in a series of papers, and they constructed extrapolation formulas to estimate the CBS limit of pair correlation energies [52-55]. This strategy was also employed by Klopper and co-workers to lower the basis-set incompleteness error (BSIE) of CCSD(T) using corrections evaluated with explicitly correlated MP2 methods [56, 57].

Concerning recent applications of FNOs to CC methods in the past decade, Krylov and co-workers extended the FNO method to the equation-of-motion CCSD treatment of open-shell systems [37, 45], DePrince and Sherrill combined FNOs with their DF-CCSD(T) implementation [58], and Aquilante and co-workers explored ideas to extrapolate toward the complete virtual basis result using FNOs [50]. Sorathia and Tew [59], motivated by Helgaker's two-point extrapolation formula [17], proposed an extrapolation approach to reduce the PNO truncation error in PNO-based local correlation methods. Their formula approximates the limit of the pair correlation energy utilizing the number of retained PNOs for a given pair of orbitals.

Very recently[51], we combined the FNO approach with the natural auxiliary function (NAF) approximation [60] and our efficient, parallel, integral-direct DF-CCSD (T) algorithm [10]. We also tested MP2 correction and extrapolation based techniques and demonstrated that the combined approaches considerably extend the reach of FNO$\operatorname{CCSD}(\mathrm{T})$. Even with relatively tight thresholds, reaction and interaction energies were obtained for molecules of 50-75 atoms with triple- and quadruple- $\zeta$ bases. However, for the largest system of $2124 \mathrm{AOs}, 65 \%$ of the FNOs were still retained with the most conservative threshold [10], while an additional order of magnitude speedup could be gained with more reliable FNO truncation corrections in combination with looser FNO thresholds.

Toward this direction, among other ideas, we build on the previous studies of Irmler and Grüneis [61, 62]. These authors utilized that the basis set convergence of the contribution of the second-order and the particle-particle ladder (PPL) terms to the CCSD correlation energy is similar [61], which is an observation going back to work of Petersson and co-workers [52-55] and was also identified in the context of CCSD's AO basis set convergence by Valeev[63]. Relying on this observation, Irmler and Grüneis proposed a multiplicative basis-set correction technique for CCSD. In their scheme, a $\triangle \mathrm{MP} 2$ correction is computed taking the difference of the MP2 correlation energy evaluated with a larger basis set and with the basis in which the CCSD energy is calculated. This $\triangle \mathrm{MP} 2$ correction is supplemented with the energy contribution of the PPL term. The latter is computed with the small basis set, but, to diminish its BSIE, is scaled by the ratio of the large- and small-basis MP2 correlation energies. 
This approach, dubbed CCSD-PPL, was found to be efficient in accelerating the basis set convergence of CCSD. However, one drawback of CCSD-PPL is that it is not size-consistent.

Another relevant development in CC theory is our perturbative triples correction, termed $(\mathrm{T}+)$, to explicitly correlated CCSD methods [64]. This approach is closely related to the $\left(\mathrm{T}^{*}\right)$ correction of Knizia et al. [65], where the $(\mathrm{T})$ contribution is scaled up in a similar way as the PPL term in the CCSD-PPL method. Just as the $\left(\mathrm{T}^{*}\right)$ correction, our $(\mathrm{T}+)$ scheme is a heuristic approach to alleviate the BSIE of $(\mathrm{T})$, but in contrast to $\left(\mathrm{T}^{*}\right)$, it is size-consistent, which is attained by scaling the contributions of individual MOs separately.

In this study, as an extension of our previous work [51], we inspect further possibilities to reduce the NO truncation error in the FNO-CCSD $(\mathrm{T})$ method. We propose a size-consistent version of the approach of Irmler and Grüneis and apply it in the NO context for molecular systems. Moreover, we also adapt our $(\mathrm{T}+)$ correction [64] to the FNO approximation to speed up the convergence of the perturbative triple excitation correction with the number of NOs. We combine these approaches with various extrapolation techniques and assess their performance in extensive benchmark calculations.

\section{Theory}

The CCSD $(\mathrm{T})$ model [5] is considered assuming a single-reference determinant. Indices $i, j, \ldots(a, b, \ldots)$ will refer to canonical occupied (virtual) orbitals, $p, q, \ldots$ will denote general orbital indices, and barred indices $\bar{a}, \bar{b}, \ldots$ will label natural orbitals of the virtual subspace.

\subsection{Frozen natural orbitals}

The NOs and the corresponding natural occupation numbers $\left(\left\{n_{a}\right\}\right)$ are computed as the eigenvectors and eigenvalues of the MP2 one-particle density matrix (OPDM):

$$
D_{a b}^{\mathrm{MP} 2}=\sum_{i j c} \frac{(c i \mid a j)(c i \mid b j)-(c i \mid a j)(b i \mid c j)}{\left(\epsilon_{i}+\epsilon_{j}-\epsilon_{a}-\epsilon_{c}\right)\left(\epsilon_{i}+\epsilon_{j}-\epsilon_{b}-\epsilon_{c}\right)} .
$$

Here, the summation runs over spin-orbital indices, $(p q \mid r s)$ is a two-electron integral in the Mulliken notation, and $\epsilon_{p}$ denotes the canonical orbital energy of orbital $p$.

In the FNO approach, only $\bar{n}_{\mathrm{v}}$ virtual NOs with the largest occupation numbers are retained for the $\operatorname{CCSD}(\mathrm{T})$ part of the calculation, i.e., the $\operatorname{CCSD}(\mathrm{T})$ amplitudes with any frozen NO index are considered to be zero. The FNO selection process assumes that the NOs with the largest occupation numbers are the most effective to span the virtual space required for the subsequent $\operatorname{CCSD}(\mathrm{T})$ calculation. For that reason, the FNO selection is governed by an occupation number threshold $(\epsilon)$. For the effective evaluation of the $(\mathrm{T})$ correction, the active virtual NOs are transformed into a semicanonical representation by diagonalizing the virtual-virtual block of the Fock matrix.

The truncation of the FNO basis set offers extensive cost-reduction over the conventional $\operatorname{CCSD}(\mathrm{T})$ approach because the operation counts required for the ratedetermining steps of both the CCSD and (T) components scale with the fourth-power of the number of virtual orbitals. Compared to the cost of an FNO-CCSD(T) compu- 
tation, the fifth-power scaling evaluation of the MP2 OPDM is usually fast and can be performed with effective DF-MP2 implementations with up to 5000 or more AOs. However, the restriction of excitations at the $\operatorname{CCSD}(\mathrm{T})$ level introduces a basis set error, for which a number of correction schemes are collected in Sect. 2.3.

\subsection{Correlation energy and its decompositions}

Let us briefly recollect the MP2, CCSD, and (T) correlation energy expressions relevant for the present discussion and refer to the literature for further details [3, 5, 10, 66-69]. The MP2 correlation energy is obtained in a spin-orbital basis as follows:

$$
E^{\mathrm{MP} 2}=\sum_{a i} \frac{f_{a i}^{2}}{\epsilon_{i}-\epsilon_{a}}+\frac{1}{4} \sum_{i j a b} \frac{[(a i \mid b j)-(a j \mid b i)]^{2}}{\epsilon_{i}+\epsilon_{j}-\epsilon_{a}-\epsilon_{b}}
$$

where $f_{a i}$ denotes the elements of the Fock matrix. Using this notation, the CCSD correlation energy in a spin-orbital basis reads as

$$
E^{\mathrm{CCSD}}=\sum_{i a} f_{a i} t_{i}^{a}+\frac{1}{4} \sum_{i j a b}[(a i \mid b j)-(a j \mid b i)] \tau_{i j}^{a b},
$$

where $\tau_{i j}^{a b}=t_{i j}^{a b}+t_{i}^{a} t_{j}^{b}-t_{i}^{b} t_{j}^{a}$ is introduced with $t_{i}^{a}$ and $t_{i j}^{a b}$ being the singles and doubles cluster amplitudes, respectively. Finally, the (T) correction can be written as

$$
E^{(\mathrm{T})}=\frac{1}{36} \sum_{i j k a b c} W_{i j k}^{a b c} t_{i j k}^{a b c}
$$

where the $t_{i j k}^{a b c}$ triple excitation amplitude and the $W_{i j k}^{a b c}$ intermediate are expressed with converged CCSD doubles amplitudes and two-electron integrals [5, 70, 71].

Based on previous experience in the literature, it is reasonable to expect that not all components of the CCSD and $(\mathrm{T})$ correlation energy converge to the complete NO basis limit at the same rate $[62,72,73]$. This motivates the introduction of different NO basis truncation corrections for terms with different convergence behavior. In the following, we explore various term specific correction ideas for three different kinds of decompositions of the $\operatorname{CCSD}(\mathrm{T})$ correlation energies.

\section{Decomposition into spin-components}

First, following Sherrill and co-workers [73], and Hobza et al. [74], the CCSD energy is rewritten as a sum of same-spin (SS) and opposite-spin (OS) contributions:

$$
E^{\mathrm{CCSD}}=\sum_{i a} f_{a i} t_{i}^{a}+E^{\mathrm{SS}-\mathrm{CCSD}}+E^{\mathrm{OS}-\mathrm{CCSD}} .
$$

The corresponding spin-orbital expressions read after spin-summation as

$$
E^{\mathrm{SS}-\mathrm{CCSD}}=\frac{1}{4} \sum_{\tilde{i} \tilde{j} \tilde{a} \tilde{b}}[(\tilde{a} \tilde{i} \mid \tilde{b} \tilde{j})-(\tilde{a} \tilde{j} \mid \tilde{b} \tilde{i})] \tau_{\tilde{i} \tilde{j}}^{\tilde{a} \tilde{b}}+\frac{1}{4} \sum_{I J A B}[(A I \mid B J)-(A J \mid B I)] \tau_{I J}^{A B},
$$


and

$$
E^{\mathrm{OS}-\mathrm{CCSD}}=\sum_{\tilde{i} J \tilde{a} B}(\tilde{a} \tilde{i} \mid B J) \tau_{\tilde{i} J}^{\tilde{a} B}
$$

where tilded lowercase (plain uppercase) indices label spin-up (spin-down) orbitals. The well-known spin components of the MP2 correlation energy are defined analogously by separating the OS and SS terms of Eq. (2) [75, 76]. The corresponding closed-shell expressions for the spin-components of both MP2 and CCSD can be found, e.g., in Ref. 73.

For clarity, empirical scaling factors characteristic of spin-component scaled methods are not employed here, as the universal or even system-specific determination of such parameters is rather problematic [73-77]. Instead, the possibilities of separate FNO corrections are explored for the OS and SS terms.

\section{Diagrammatic correlation energy decomposition}

In a separate line of thought, Irmler and Grüneis [61,62] proposed a diagrammatic decomposition of the CCSD correlation energy:

$$
E^{\mathrm{CCSD}}=E^{\mathrm{MP} 2}+E^{\mathrm{PPL}}+E^{\mathrm{rest}},
$$

where $E^{\mathrm{MP} 2}$ is the MP2 correlation energy of Eq. (2). The second term, $E^{\mathrm{PPL}}$ is the correlation energy contribution assigned to the PPL diagram:

$$
E^{\mathrm{PPL}}=\frac{1}{8} \sum_{i j a b} \frac{(a i \mid b j)-(a j \mid b i)}{\epsilon_{i}+\epsilon_{j}-\epsilon_{a}-\epsilon_{b}} \sum_{c d}[(a c \mid b d)-(a d \mid b c)] \tau_{i j}^{c d}=\frac{1}{4} \sum_{i j a b} T_{i j}^{a b} A_{i j}^{a b},
$$

where the MP1 amplitudes $\left(T_{i j}^{a b}\right)$ are contracted with the PPL term of the CCSD amplitude equations, denoted as $A_{i j}^{a b}$. Finally, for the present purposes, the remaining terms of the CCSD correlation energy are collected into $E^{\text {rest }}=E^{\mathrm{CCSD}}-E^{\mathrm{MP} 2}-E^{\mathrm{PPL}}$.

\section{Orbital and orbital pair specific energy contributions}

Ultimately, the correlation energy can be decomposed into individual orbital or even orbital pair contributions, similarly to the formulation of local correlation methods [12-15]. One common feature of recent local correlation methods is the compression of the MO basis using orbital or orbital pair specific NOs. For instance, in our LNO family of methods $[12,48,69,78-82]$, all of the above correlation energy expressions can be decomposed into electron (or orbital) specific contributions:

$$
E^{\mathrm{M}}=\sum_{i} \delta E_{i}^{\mathrm{M}}
$$

where method M can refer to MP2 [80-82], CCSD [48, 79], (T) [12, 48, 69], or even higher orders of $\mathrm{CC}$ theory $[48,78]$. For instance, for $\mathrm{M}=\mathrm{MP} 2$, the correlation energy 
of Eq. (2) can be recast as

$$
E^{\mathrm{MP} 2}=\sum_{i}\left(\sum_{a} \frac{f_{a i}^{2}}{\epsilon_{i}-\epsilon_{a}}+\frac{1}{4} \sum_{j a b} \frac{[(a i \mid b j)-(a j \mid b i)]^{2}}{\epsilon_{i}+\epsilon_{j}-\epsilon_{a}-\epsilon_{b}}\right)=\sum_{i} \delta E_{i}^{\mathrm{MP} 2}
$$

in order to define the $\delta E_{i}^{\mathrm{MP} 2}$ contribution of orbital $i$. Similarly, the $\delta E_{i}^{\mathrm{CCSD}}$ and $\delta E_{i}^{(\mathrm{T})}$ correlation energy contributions are obtained by pulling out the summation over orbital index $i$ in Eqs. (3) and (4), respectively. Similarly, pair correlation energy contributions are obtained by separating two occupied summation indices in Eqs. (2)(4). For instance, for $\mathrm{M}=\mathrm{MP} 2$, the corresponding pair correlation energy is defined as

$$
\delta E_{i j}^{\mathrm{MP} 2}=\frac{1}{2} \sum_{a b} \frac{[(a i \mid b j)-(a j \mid b i)]^{2}}{\epsilon_{i}+\epsilon_{j}-\epsilon_{a}-\epsilon_{b}},
$$

and $\delta E_{i j}^{\mathrm{CCSD}}$ and $\delta E_{i j}^{(\mathrm{T})}$ are obtained analogously.

Naturally, the above energy decomposition schemes can also be combined. For instance, in Sect. 3, we analyze separately the spin-components of the MP2, PPL, and remaining CCSD terms. Additionally, the separation of the SS and OS terms is combined with the orbital specific correlation energy decomposition. In principle, the separate spin-components of all diagrammatic terms can be studied for each orbital or orbital pair, but this excessive decomposition is not motivated by our results collected below.

\subsection{Correction schemes for the FNO truncation error}

For discussing the relation between the FNO truncation error and the corresponding FNO occupation number threshold, $\epsilon$, let us (formally) parametrize the correlation energy as a function of the FNO threshold:

$$
E_{\epsilon}^{\mathrm{M}}=E_{\epsilon=0}^{\mathrm{M}}+C^{\mathrm{M}} f^{\mathrm{M}}(\epsilon),
$$

where $E_{\epsilon}^{\mathrm{M}}$ denotes the correlation energy obtained with method M in the FNO basis defined by $\epsilon$. In general, constant $C^{\mathrm{M}}$ and function $f^{\mathrm{M}}(\epsilon)$ depend on the $\mathrm{M}$ method, and their exact form is unknown for either MP2 or CC methods. The common idea behind the following FNO truncation correction schemes is to make assumptions on $C^{\mathrm{M}}$ and $f^{\mathrm{M}}(\epsilon)$ leading to a low-cost estimate of the missing $E_{\epsilon=0}^{\mathrm{M}}-E_{\epsilon}^{\mathrm{M}}$ portion of the $\mathrm{M}=\mathrm{CCSD}$, $(\mathrm{T})$, etc. correlation energy.

MP2-based additive or focal-point correction ( $\triangle M P 2)$

Assuming that $f^{\mathrm{M}}(\epsilon)=f^{\mathrm{MP} 2}(\epsilon)$ for $\mathrm{M}=\mathrm{CCSD}, \operatorname{CCSD}(\mathrm{T})$, etc. leads to the following formula for the MP2-based additively corrected correlation energy, $E^{\mathrm{M} \Delta}$ :

$$
E_{\epsilon=0}^{\mathrm{M}} \approx E^{\mathrm{M} \Delta}=E_{\epsilon}^{\mathrm{M}}+\frac{C^{\mathrm{M}}}{C^{\mathrm{MP} 2}}\left(E_{\epsilon=0}^{\mathrm{MP} 2}-E_{\epsilon}^{\mathrm{MP} 2}\right)=E_{\epsilon}^{\mathrm{M}}+F_{\epsilon}^{\Delta \mathrm{MP} 2} \Delta E_{\epsilon}^{\mathrm{MP} 2},
$$


where usually $F_{\epsilon}^{\Delta \mathrm{MP} 2}=\frac{C^{\mathrm{M}}}{C^{\mathrm{MP} 2}}=1$ is employed for the unknown $F_{\epsilon}^{\Delta \mathrm{MP} 2}$. However, trivial algebra results in a different form for the optimal scaling factor, $F_{\epsilon}^{\text {opt }}$, of the $\Delta E_{\epsilon}^{\mathrm{MP} 2}$ energy correction:

$$
E_{\epsilon=0}^{\mathrm{M}}=E_{\epsilon}^{\mathrm{M}}+\frac{E_{\epsilon=0}^{\mathrm{M}}-E_{\epsilon}^{\mathrm{M}}}{E_{\epsilon=0}^{\mathrm{MP} 2}-E_{\epsilon}^{\mathrm{MP} 2}} \Delta E_{\epsilon}^{\mathrm{MP} 2}=E_{\epsilon}^{\mathrm{M}}+F_{\epsilon}^{\mathrm{opt}} \Delta E_{\epsilon}^{\mathrm{MP} 2} .
$$

While this form of $F_{\epsilon}^{\mathrm{opt}}$ is not practical because of its $E_{\epsilon=0}^{\mathrm{M}}$ dependence, it will provide useful insight below. Obviously, in case of $F_{\epsilon}^{\Delta \mathrm{MP} 2}=1$, the decomposition of $E_{\epsilon}^{\mathrm{M}}$ does not bring any advantages, but it appears to be beneficial to search for better $F_{\epsilon}^{\text {opt }}$ approximations for separate energy components.

\section{MP2-based multiplicative corrections}

An approximation to $F_{\epsilon}^{\text {opt }}$ as

$$
F_{\epsilon}^{\mathrm{opt}}=\frac{E_{\epsilon=0}^{\mathrm{M}}-E_{\epsilon}^{\mathrm{M}}}{E_{\epsilon=0}^{\mathrm{MP} 2}-E_{\epsilon}^{\mathrm{MP} 2}} \approx F_{\epsilon}^{*}=\frac{E_{\epsilon}^{\mathrm{M}}}{E_{\epsilon}^{\mathrm{MP} 2}}
$$

leads to the commonly employed multiplicative correction of correlation energies:

$$
E^{\mathrm{M}^{*}}=E_{\epsilon}^{\mathrm{M}}+F_{\epsilon}^{*} \Delta E_{\epsilon}^{\mathrm{MP} 2}=E_{\epsilon}^{\mathrm{M}}+\frac{E_{\epsilon}^{\mathrm{M}}}{E_{\epsilon}^{\mathrm{MP} 2}} \Delta E_{\epsilon}^{\mathrm{MP} 2}=\frac{E_{\epsilon=0}^{\mathrm{MP} 2}}{E_{\epsilon}^{\mathrm{MP} 2}} E_{\epsilon}^{\mathrm{M}},
$$

where the truncated $E_{\epsilon}^{\mathrm{M}}$ is simply scaled by the ratio of the MP2 correlation energies in the complete and truncated NO bases, $\frac{E_{\epsilon 0}^{\mathrm{MP} 2}}{E_{\mathrm{MP} 2}^{\mathrm{MP}}}$.

Two scaling techniques of this kind are known in the context of AO BSIE correction $[62,65]$. First, in the $\left(\mathrm{T}^{*}\right)$ method, introduced by Knizia, Adler, and Werner (KAW) [65], the (T) correlation energy component is scaled by the ratio of the MP2-F12 and MP2 correlation energies to correct for the BSIE of (T). A related alternative, suggested by Peterson et al., scales the (T) energies by the ratio of CCSD-F12 and CCSD correlation energies[83]. Recently, we proposed a similar approach utilizing orbital or orbital pair energy decomposition ideas in order to eliminate the size-inconsistency of $\left(\mathrm{T}^{*}\right)$ [64]. The resulting $(\mathrm{T}+)$ scheme successfully retains the BSIE reduction properties of $\left(\mathrm{T}^{*}\right)$ also for systems that are sensitive to size-consistency errors [64]. Note that scaling of the $(\mathrm{T})$ energies using the same scaling factor for all species, e.g., by the factor determined for the largest species [13] or via empirical methods [83], is also size-consistent, but not employed here.

Motivated by these results, here, we investigate analogous ideas to our $(\mathrm{T}+) \mathrm{BSIE}$ correction in order to reduce the FNO error of the (T) correction, because, to our knowledge, the direct correction of $(\mathrm{T})$ was not yet explored in the FNO context. The corresponding $\left(\mathrm{T}^{*}\right)$ correlation energy is defined as

$$
E^{\left(\mathrm{T}^{*}\right)}=\frac{E_{\epsilon=0}^{\mathrm{MP} 2}}{E_{\epsilon}^{\mathrm{MP} 2}} E_{\epsilon}^{(\mathrm{T})},
$$

while orbital specific scaling factors of $q_{i}=\frac{\delta E_{i, \epsilon=0}^{\mathrm{MP} 2}}{\delta E_{i, \epsilon}^{\mathrm{MP} 2}}$ are introduced for the size-consistent $(\mathrm{T}+)$ variant. Considering the $(\mathrm{T})$ correlation energy expression of Eq. (4), as it was 
demonstrated in Ref. 64 , it is more beneficial to introduce the $(\mathrm{T}+)$ method in the following form:

$$
E^{(\mathrm{T}+)}=\frac{1}{3}\left(\sum_{i} q_{i} E_{i, \epsilon}^{(\mathrm{T})}+\sum_{j} q_{j} E_{j, \epsilon}^{(\mathrm{T})}+\sum_{k} q_{k} E_{k, \epsilon}^{(\mathrm{T})}\right)=\frac{1}{36} \sum_{i j k \bar{a} \bar{b} \bar{c}} \frac{q_{i}+q_{j}+q_{k}}{3} W_{i j k}^{\bar{a} \bar{b} \bar{c}} t_{i j k}^{\bar{a} \bar{b} \bar{c}} .
$$

Since the orbital contributions are scaled separately with an orbital specific factor, the $(\mathrm{T}+)$ correction is clearly size-consistent. Moreover, a more balanced performance can be expected because the FNO truncation correction is decomposed into orbital specific contributions. Finally, a pair contribution based form, more suitable for paircorrelation theories, can also be defined via the pair correlation energy scaling quotient, $q_{i j}=\frac{\delta E_{i j, \epsilon=0}^{\mathrm{MP} 2}}{\delta E_{i j, \epsilon}^{\mathrm{MP2}}}$ :

$$
E^{(\mathrm{T}+)^{\prime}}=\frac{1}{36} \sum_{i j k \bar{a} \bar{b} \bar{c}} \frac{q_{i j}+q_{i k}+q_{j k}}{3} W_{i j k}^{\bar{a} \bar{b} \bar{c}} t_{i j k}^{\bar{a} \bar{b} \bar{c}}
$$

The second multiplicative correction considered here is connected to the diagrammatic decomposition of the CCSD correlation energy. Recently, Irmler and Grüneis $[61,62]$ recognized that the convergence of the PPL term, defined by Eq. (9), with the AO basis completeness is similar to that of the MP2 term but of opposite sign. This observation was exploited to correct for the BSIE of the PPL term. More precisely, the same scaling factor employed in the $\left(\mathrm{T}^{*}\right)$ method, namely the ratio of the MP2/CBS and the MP2 correlation energy of the given AO basis was utilized [62]. Here, we employ this formula for the correction of the FNO error within the PPL term. Due to the similarities to the $\left(\mathrm{T}^{*}\right)$ method as well as to the AO BSIE correction of PPL in Ref. 62 , this scheme is labeled here as the $\triangle \mathrm{PPL}^{*}$ correction:

$E^{\mathrm{CCSD}-\mathrm{PPL}^{*}}=E_{\epsilon}^{\mathrm{CCSD}}+\Delta E_{\epsilon}^{\mathrm{MP} 2}+\left(\frac{E_{\epsilon=0}^{\mathrm{MP} 2}}{E_{\epsilon}^{\mathrm{MP} 2}}-1\right) E_{\epsilon}^{\mathrm{PPL}}=E_{\epsilon}^{\mathrm{CCSD}}+\Delta E_{\epsilon}^{\mathrm{MP} 2}+\Delta E_{\epsilon}^{\mathrm{PPL}}{ }^{*}$

Here, the $\Delta E_{\epsilon}^{\mathrm{MP} 2}$ additive correction of Eq. (14) eliminates the FNO truncation error of the MP2 term, while the $\Delta E_{\epsilon}^{\mathrm{PPL}}{ }^{*}$ term corrects for the truncation error of the PPL contribution. As in the original proposition of Ref. 62 , the $\Delta \mathrm{PPL}^{*}$ correction does not improve on the FNO error of the $E^{\text {rest }}$ term of Eq. (8) since the basis set truncation error of $E^{\text {rest }}$ is often much smaller than that of the MP2 and PPL terms.

Analogously to the case of the $\left(\mathrm{T}^{*}\right)$ method, the $\Delta \mathrm{PPL}^{*}$ correction is not sizeconsistent either. The size-consistency error of $\left(\mathrm{T}^{*}\right)$ is usually small, but it may reach up to $1 \mathrm{kcal} / \mathrm{mol}$ under unfortunate circumstances [64]. Moreover, the sizeinconsistency of $\triangle \mathrm{PPL}^{*}$ could be even more pronounced, because the PPL correlation energy contribution is about an order of magnitude larger than the (T) correction. Therefore, we also propose a size-consistent PPL correction, denoted as $\triangle \mathrm{PPL}+$ :

$$
\Delta E_{\epsilon}^{\mathrm{PPL}+}=\frac{1}{2}\left(\sum_{i}\left(q_{i}-1\right) E_{i, \epsilon}^{\mathrm{PPL}}+\sum_{j}\left(q_{j}-1\right) E_{j, \epsilon}^{\mathrm{PPL}}\right)=\sum_{i j \bar{a} \bar{b}} \frac{q_{i}+q_{j}-2}{8} T_{i j}^{\bar{a} \bar{b}} A_{i j}^{\bar{a} \bar{b}},
$$

which can replace $\Delta E_{\epsilon}^{\mathrm{PPL}^{*}}$ in Eq. (21) above to define $E^{\mathrm{CCSD}-\mathrm{PPL}+}$. The analogous 
expression, more suitable for electron-pair theories is defined as

$$
\Delta E_{\epsilon}^{\mathrm{PPL}+^{\prime}}=\sum_{i j \bar{a} \bar{b}} \frac{q_{i j}-1}{4} T_{i j}^{\bar{a} \bar{b}} A_{i j}^{\bar{a} \bar{b}} .
$$

\section{Extrapolation schemes}

A closer inspection of the $F_{\epsilon}^{\text {opt }} \approx F_{\epsilon}^{*}$ approximation in Eq. (16) leads to an alternative line of FNO corrections. The $\frac{E_{\epsilon=0}^{\mathrm{M}}-E_{\epsilon}^{\mathrm{M}}}{E_{\epsilon=0}^{\mathrm{MP}}-E_{\epsilon}^{\mathrm{MP} 2}}$ quotient can be interpreted as the ratio of the correlation energies missing from the FNO-M and FNO-MP2 computations, respectively. Compared to that, the practical $F_{\epsilon}^{*}$ holds the ratio of the correlation energies retained in the FNO-M and FNO-MP2 calculations, and thus appears to be suboptimal.

From an other perspective, $\frac{E_{\epsilon=0}^{\mathrm{M}}-E_{\epsilon}^{\mathrm{M}}}{E_{\epsilon=0}^{\mathrm{M} 2}-E_{\epsilon}^{\mathrm{MP} 2}}$ is a difference quotient for the $E_{\epsilon}^{\mathrm{M}}\left(E_{\epsilon}^{\mathrm{MP} 2}\right)$ function evaluated at the $\epsilon=0$ and $\epsilon$ points. While $F_{\epsilon}^{*}=\frac{E_{\epsilon}^{\mathrm{M}}-E_{\epsilon}^{\mathrm{M}}}{E_{\epsilon}^{\mathrm{MP} 2}-E_{\epsilon=\infty}^{\mathrm{MP}}}$ formally has the same difference quotient form, it is evaluated at the $\epsilon$ and $\epsilon=\infty$ points, where $\epsilon=\infty$ denotes the case of zero retained FNOs. Consequently, $F_{\epsilon}^{*}$ can only be ideal if the slope of the $E_{\epsilon}^{\mathrm{M}}\left(E_{\epsilon}^{\mathrm{MP} 2}\right)$ function is the same at the $[\epsilon=\infty, \epsilon]$ and the $[\epsilon, \epsilon=0]$ intervals. This, however, cannot be expected since $f^{\mathrm{MP} 2}(\epsilon)=f^{\mathrm{M}}(\epsilon)$ is not a good assumption in the case of $\mathrm{M}=\mathrm{CCSD}, \operatorname{CCSD}(\mathrm{T})$, etc. for all $\epsilon$ values. In other words, $E_{\epsilon}^{\mathrm{M}}\left(E_{\epsilon}^{\mathrm{MP} 2}\right)$ is generally non-linear in $\epsilon$, especially for $\epsilon$ values far from $\epsilon=0$.

The above analysis suggests a better approximation to $F_{\epsilon}^{\mathrm{opt}}$. To that end, an estimator of the ideal difference quotient should be evaluated close to the $\epsilon=0$ point, i.e., at a region where the linearity of $E_{\epsilon}^{\mathrm{M}}\left(E_{\epsilon}^{\mathrm{MP} 2}\right)$ is a reasonable assumption. Hence we suggest to perform a second FNO-M computation at a somewhat looser threshold than $\epsilon$, denoted as $\epsilon^{\prime}$, and to obtain an $F_{\epsilon}^{\text {opt }}$ estimate as

$$
F_{\epsilon}^{\mathrm{opt}} \approx F_{\epsilon, \epsilon^{\prime}}^{\mathrm{LE}}=\frac{E_{\epsilon}^{\mathrm{M}}-E_{\epsilon^{\prime}}^{\mathrm{M}}}{E_{\epsilon}^{\mathrm{MP} 2}-E_{\epsilon^{\prime}}^{\mathrm{MP} 2}} .
$$

Because of the above assumptions this approach is equivalent to the linear extrapolation (LE) of $E_{\epsilon}^{\mathrm{M}}\left(E_{\epsilon}^{\mathrm{MP} 2}\right)$ with respect to $E^{\mathrm{MP} 2}$, denoted as LEMP2:

$$
E^{\mathrm{M} / \mathrm{LEMP} 2}=E_{\epsilon}^{\mathrm{M}}+F_{\epsilon, \epsilon^{\prime}}^{\mathrm{LE}} \Delta E_{\epsilon}^{\mathrm{MP} 2}=E_{\epsilon}^{\mathrm{M}}+\frac{E_{\epsilon}^{\mathrm{M}}-E_{\epsilon^{\prime}}^{\mathrm{M}}}{E_{\epsilon}^{\mathrm{MP} 2}-E_{\epsilon^{\prime}}^{\mathrm{MP} 2}}\left(E_{\epsilon=0}^{\mathrm{MP} 2}-E_{\epsilon}^{\mathrm{MP} 2}\right),
$$

where we extrapolate to the known $E_{\epsilon=0}^{\mathrm{MP} 2}$ point using the values of $E_{\epsilon}^{\mathrm{M}}\left(E_{\epsilon}^{\mathrm{MP} 2}\right)$ evaluated at the $\epsilon$ and $\epsilon^{\prime}$ points. Note that adding more data points $\left(\epsilon^{\prime \prime}, \ldots\right)$ could help to take into account the non-linearity of $E_{\epsilon}^{\mathrm{M}}\left(E_{\epsilon}^{\mathrm{MP} 2}\right)$ around $\epsilon$. For that purpose, sequence transformations, such as the Shanks or the Richardson extrapolations, have been assessed [50, 51], but they did not lead to substantial improvements over the performance of the linear extrapolation for practical $\epsilon$ values.

Again, the energy decomposition schemes considered here allow for the introduction of termwise linear extrapolation based corrections. For instance, we find particularly interesting and investigate the separate extrapolation of the OS-CCSD and SS-CCSD terms, as well as the spin components of the PPL and the remaining CCSD terms as a function of OS-MP2 and SS-MP2. Additionally, the separate extrapolation of CCSD and $(\mathrm{T})$ allows for the combination of linear extrapolation for one and, for 
instance, additive or multiplicative corrections for the other term. Furthermore, the introduction of orbital specific energy decomposition ideas lead to a size-consistent linear extrapolation based correction:

$$
E^{\mathrm{M} / \mathrm{SC}-\mathrm{LEMP} 2}=E_{\epsilon}^{\mathrm{M}}+\sum_{i} F_{i ; \epsilon, \epsilon^{\prime}}^{\mathrm{LE}} \Delta E_{i, \epsilon}^{\mathrm{MP} 2}=E_{\epsilon}^{\mathrm{M}}+\sum_{i} \frac{E_{i, \epsilon}^{\mathrm{M}}-E_{i, \epsilon^{\prime}}^{\mathrm{M}}}{E_{i, \epsilon}^{\mathrm{MP} 2}-E_{i, \epsilon^{\prime}}^{\mathrm{MP} 2}} \Delta E_{i, \epsilon}^{\mathrm{MP} 2} .
$$

Finally, let us note that correlation energy extrapolations have been performed previously in the FNO context using different independent variables. For instance, the occupation number threshold (ONT) itself was employed [37] in this role, and later, an occupation number based variable was also suggested [50]. The latter, cumulative occupation number threshold (COT) [50], $\zeta$ is defined as:

$$
\zeta=\frac{\sum_{a}^{n_{\mathrm{a}}>\epsilon} n_{a}}{\operatorname{Tr}\left(\mathbf{D}^{\mathrm{MP} 2}\right)}
$$

There is a one-to-one correspondence between ONT and COT, e.g., the complete FNO basis limit is defined by $\zeta=1$ or $\epsilon=0$. Recently, we demonstrated the excellent correlation of the ONT and COT variables [51], thus here, we focus on extrapolations as a function of only one of them, namely COT. While previous COT approaches extrapolated the total correlation energy, here, we also explore the possibilities to extrapolate, e.g., the PPL, remaining CCSD, or (T) separately as a function of COT.

We note in passing that the correlation energy decomposition and NO basis set correction ideas considered here could also be employed to correct for AO basis set incompleteness. While explicitly correlated schemes and basis set extrapolation formulae are both effective to accelerate the convergence with the AO basis set, the analogues of neither the F12 approaches nor the CBS extrapolations are available to correct for FNO truncations. For that reason, here, we focus on the correction for discarded FNOs, while the application of the presented ideas for the improvement on AO basis set incompleteness will be explored elsewhere.

\section{Results and discussion}

\subsection{Computational details}

The present approaches have been implemented in the MRCC suite of quantum chemical programs $[84,85]$, which is also used in all the calculations. While, for brevity, Sect. 2 collects the spin-orbital expressions, the corresponding spatial-orbital based equations have also been derived and implemented in our closed-shell DF-MP2 [80] and DF$\operatorname{CCSD}(\mathrm{T})[10]$ programs. For both open- and closed-shell cases, the complete virtual orbital as well as the FNO computations were performed with the hand-optimized, parallel and (partially) integral-direct DF-CCSD(T) implementations of the MrCC suite $[10,51,85]$.

As the AO basis set, the correlation consistent $X$-tuple- $\zeta$ (aug-)cc-pVXZ sets [86, 87] as well as its cc-pV $(X+\mathrm{d}) \mathrm{Z}$ extensions [88] were employed with the corresponding DF auxiliary bases, (aug-)cc-pVXZ-RI-JK [89] and (aug-)cc-pVXZ-RI [90]. The core electrons were frozen in all correlation calculations. Unlike to our previous study [51], the NAF approximation was not employed here for simplicity. 
For benchmarking, the closed-shell reaction energy test set developed by Adler and Werner (AW) [91], as well as the closed- and open-shell reaction energy and atomization energy test sets of KAW [65] were taken. The AW test set contains 58 molecules with up to 18 atoms forming 51 reactions. The KAW compilation incorporates 66 atoms and molecules built of first- and second-row elements, which form 48 open-shell reactions, 28 closed-shell reactions, and 49 atomization processes. Only the species and structures were taken from Refs. 91 and 65. Non-covalent interactions are assessed on the 24 interaction energies of the A24 test set[92]. The conventional $\operatorname{CCSD}(\mathrm{T})$ reference energies for the AW set was recomputed in our previous work [51], and references for the species in the KAW and A24 sets were recomputed for the present study in order to avoid inconsistencies. To characterize the performance of the methods, the mean absolute error (MAE), mean signed error (MSE), the standard deviation (STD), and the maximum error (MAX) of the computed results will be applied. Throughout this work, LEMP2 as well as ONT/COT extrapolated results reported for $\epsilon=10^{-x}$ are obtained using $\epsilon=10^{-x}$ and $\epsilon^{\prime}=10^{-x+0.5}$ data points.

\subsection{Correlation energies of closed-shell molecules}

Since there are a large number of methods collected in Sect. 2, especially for CCSD, our first goal is to compare their performance on the correlation energies of the somewhat larger species in the AW compilation. Then the promising approaches for CCSD, as well as the somewhat smaller number of $(\mathrm{T})$ correction ideas will be analyzed further in Sect. 3.3. As it will be important to keep track of the error compensations of the various terms, the average of the signed relative correlation energy errors are collected in Table 1 with the cc-pVQZ basis set using the exact CCSD correlation energies as reference. As noted in our previous report, the FNO thresholds suitable for quadruple$\zeta$ basis sets lead to negligible truncation errors with triple- $\zeta$ bases [51], thus here, we focus our investigation on the former.

The uncorrected relative errors of $-0.4 \%$ to $-4 \%$ for the total CCSD energies are quite large with the selected $\epsilon=10^{-5}-10^{-4}$ thresholds (first row of Table 1). The comparison of the PPL and rest of CCSD errors of Table 1 to these total CCSD deviation shows that the MP2 component is indeed dominant, followed by the 2-3 times smaller PPL errors with opposite sign. The errors for the rest of the CCSD components exhibit an additional drop of a factor of 3-5 compared to those of PPL. Considering the correlation energy composition of about $98 \pm 3 \%,-25 \pm 2 \%$, and $27 \pm 4 \%$ (in the form of average \pm STD) for the MP2, PPL, and rest of CCSD terms of these closed-shell systems, respectively, the convergence of the MP2 and PPL terms exhibit a similar pattern which is slower than that of the remaining CCSD terms.

Further decomposition of these uncorrected error components to OS and SS terms are gathered in rows 2 and 3 of Table 1. For all three CCSD terms (MP2, PPL, and rest) the SS component converges significantly faster, thus about $90 \%$ of the truncation errors can be attributed to the OS contributions. Compared to the quite representative $80 \%$ to $20 \%$ ratio of the OS and SS correlation energy components, the smaller SS errors are not completely the results of the smaller size of the SS contributions. An additional factor is that the problematic electron-electron cusps cause slower basis set convergence in the OS component of the wave function than in the SS component.

The simplest $\triangle \mathrm{MP} 2$ correction (row 4) eliminates the largest source of error originating from the MP2 term. Its relatively good performance can thus be attributed to the systematic compensation of the negative PPL and the positive $E^{\text {rest }}$ errors. 
Table 1. Mean (signed) relative correlation energy errors (in \%) compared to the untruncated CCSD total correlation energy as a function of the $\epsilon$ FNO threshold. See Sect. 2 and the discussion of the table for the definition of various method types.

\begin{tabular}{l|ccc|ccc|ccc}
\hline \hline & \multicolumn{3}{|c|}{ Total CCSD } & \multicolumn{3}{c|}{ PPL } & \multicolumn{3}{c}{ Rest of CCSD } \\
FNO threshold, $\epsilon:$ & $10^{-4}$ & $10^{-4.5}$ & $10^{-5}$ & $10^{-4}$ & $10^{-4.5}$ & $10^{-5}$ & $10^{-4}$ & $10^{-4.5}$ & $10^{-5}$ \\
\hline Uncorrected & -4.0 & -1.3 & -0.39 & 2.1 & 0.85 & 0.28 & -0.71 & -0.18 & -0.04 \\
OS uncorrected & -3.5 & -1.2 & -0.36 & 1.9 & 0.79 & 0.27 & -0.61 & -0.16 & -0.03 \\
SS uncorrected & -0.48 & -0.14 & -0.03 & 0.17 & 0.06 & 0.01 & -0.10 & -0.03 & -0.01 \\
$\Delta$ MP2 & 1.4 & 0.66 & 0.24 & 2.1 & 0.87 & 0.28 & -0.71 & -0.18 & -0.04 \\
$F_{\epsilon}^{*}=\frac{E_{\epsilon}^{\mathrm{M}}}{E_{\epsilon}^{\mathrm{MP} 2}}$ scaled & 1.6 & 0.75 & 0.27 & 0.74 & 0.33 & 0.11 & 0.85 & 0.42 & 0.16 \\
$\Delta$ PPL (incl. $\Delta \mathrm{MP2})$ & 0.03 & 0.14 & 0.08 & 0.74 & 0.33 & 0.11 & -0.71 & -0.18 & -0.04 \\
LEMP2 & - & 0.20 & 0.044 & - & 0.08 & 0.017 & - & 0.12 & 0.027 \\
OS LEMP2 & - & 0.19 & 0.040 & - & 0.07 & 0.015 & - & 0.12 & 0.020 \\
SS LEMP2 & - & 0.006 & 0.001 & - & 0.01 & 0.001 & - & -0.003 & 0.000 \\
ONT extrapolation & - & -0.11 & 0.05 & - & 0.28 & 0.02 & - & 0.06 & 0.031 \\
COT extrapolation & - & -0.10 & 0.09 & - & 0.26 & 0.001 & - & 0.06 & 0.036 \\
\hline \hline
\end{tabular}

According to Eq. (17), multiplying the CCSD energy by $F_{\epsilon}^{*}=\frac{E_{\epsilon}^{\mathrm{M}}}{E_{\epsilon}^{\mathrm{MP2}}}$ (row 5 ) is also exact for the MP2 term and was also suggested to reduce the error in the PPL term [62]. Indeed, compared to the uncorrected PPL results, the scaled PPL errors improve by a factor of 2.5-3 and retain their positive sign. Interestingly, upon scaling with $F^{*}$, the errors in $E^{\text {rest }}$ increase compared to the uncorrected ones, and more importantly, their sign is flipped. This explains the observation that the multiplicatively corrected total CCSD energies are worse than the $\triangle \mathrm{MP} 2$ corrected ones even if the former are theoretically more promising to improve on both dominant sources of error (MP2 and PPL). From this perspective the excellent performance of $\triangle$ PPL can also be understood (row 6): it eliminates the MP2 error and shrinks the PPL error to the size of the uncorrected errors of $E^{\text {rest }}$, which two then cancel each other almost completely. This fortunate behavior of the $\triangle \mathrm{PPL}$ correction was also documented by Irmler and Grüneis in the context of AO BSIE reduction [61,62].

Since the cancellation of the multiplicatively corrected PPL and uncorrected $E^{\text {rest }}$ error might not be universal, there is still room for improvement in these error components. Thus, rows 7-9 of Table 1 also collect the results of linear extrapolation of the total CCSD, OS-CCSD, and SS-CCSD energies as a function of the MP2, OSMP2, and SS-MP2 correlation energies, respectively. Similar to the $\triangle \mathrm{MP} 2$ and $\triangle \mathrm{PPL}$ approaches, linear extrapolation with respect to $E^{\mathrm{MP} 2}$ also completely eliminates the error of the MP2 term. More importantly, in accord with the expectation that the LEMP2 scaling factor in Eq. (24) is closer to the optimal one, the PPL errors of LEMP2 drop by an order of magnitude (factor of 4-5) compared to the uncorrected ( $\triangle$ PPL corrected) PPL errors. Some improvement is also observed for the LEMP2 corrected $E^{\text {rest }}$ term, but its errors change sign, and thus the beneficial error cancellation with the remaining PPL error is lost also for LEMP2. The spin-component decomposition for LEMP2 shows that the FNO errors of all three types of SS components can be practically eliminated using LEMP2. Again, the LEMP2 errors are dominated by the OS components, and only marginal benefits arose from the separate extrapolation of the spin components. For the tested systems, the difference of LEMP2 and its sizeconsistent variant is negligible, thus the above statements hold for both, and we will not discuss them separately for the rest of this work.

Finally, the last two rows of Table 1 show the similar performance of the ONT and COT extrapolations. They perform comparably to $\triangle \mathrm{PPL}$ for the PPL term, at least in terms of the MSE, and bring the most notable improvement for $E^{\text {rest }}$. The signed average errors of ONT and COT also appear to be competitive for CCSD. 
However, their corresponding STD and maximum error measures (e.g., $0.34 \%$ and $2.4 \%$, respectively, for COT with $\epsilon=10^{-4.5}$ ) show that the good MSE values result from balanced positive and negative correlation energy errors. For that reason, ONT/COT extrapolations appear to be most promising for the improvement of the $E^{\text {rest }}$ term.

In order to simplify the remaining numerical analysis, the discussion of the spincomponent as well as the orbital pair decomposition alternatives is limited to the AW set. Although an improvement can be achieved by separating the OS and SS components, it is mostly small due to the faster convergence of the SS errors and the smaller SS components of the remaining errors. This also holds when the spin components of $\triangle \mathrm{PPL}$ are separated. For instance, scaling with the corresponding OS and SS MP2 energy ratios, the OS $\triangle \mathrm{PPL}$ and SS $\triangle \mathrm{PPL}$ errors are $0.58 \%$ and $0.08 \%$, respectively, with $\epsilon=10^{-4}$. The sum of these is, on the one hand, a bit smaller than the corresponding $0.74 \%$ value of $\triangle \mathrm{PPL}$, but the OS/SS separation worsens the error compensation with the $E^{\text {rest }}$ error. The decomposition of the PPL components (either total PPL or OS and SS) into orbital or orbital pair terms for the size-consistent scaling does not have a notable effect, at least for this test set. For instance, the deviation between the relative error in the total and OS PPL terms with the $\triangle \mathrm{PPL}^{*}$, $\triangle \mathrm{PPL}+$, or $\Delta \mathrm{PPL}+^{\prime}$ variants is below $0.02 \%$ with $\epsilon=10^{-4.5}$ and even smaller for the SS component or with $\epsilon=10^{-5}$.

To gain a better understanding of the OS error components, we also looked at its diagonal $\left(\sum_{i} \delta E_{i i}^{\mathrm{OS}-\mathrm{M}}\right)$ and off-diagonal $\left(\sum_{i \neq j} \delta E_{i j}^{\mathrm{OS}-\mathrm{M}}\right)$ components motivated by costeffective correction possibilities for the former. To cut the long story short, the relative error contribution of the diagonal components is indeed higher than the relative number of terms in the above sums, but the errors of the off-diagonal terms are still about 6 (3) times larger for the PPL (rest of CCSD) contributions than those of the diagonal terms.

\subsection{Correlation energies of closed- and open-shell species}

Since some of the above methods benefit from compensation of errors, it is also interesting to explore their behavior on a wider range of molecules including also more challenging open-shell species. For that end, the correlation energy errors are also considered for the KAW compilation using cc-pV(Q+d)Z basis set. A notable difference with the previous closed-shell AW test set is the much less consistent correlation energy composition in the KAW set. Since $94 \pm 7 \%,-25 \pm 16 \%$, and $34 \pm 13 \%$ of the CCSD correlation energy is assigned to the MP2, PPL, and rest of CCSD terms, respectively, the above error compensation trends could be less systematic.

The three panels of Fig. 1 collect relative signed error averages with respect to the exact reference separately for the PPL, $E^{\text {rest }}$, and $(\mathrm{T})$ terms. To avoid misinterpretations due to potential cancellation of positive and negative errors in the signed averages, the corresponding STD values are also represented by the error bars of Fig. 1. The $\triangle \mathrm{PPL}^{*}$ and $\triangle \mathrm{PPL}+$, the $\left(\mathrm{T}^{*}\right)$ and $(\mathrm{T}+)$, the LEMP2 and its separate spin-component variant, as well as the COT and ONT methods would be practically indistinguishable in these plots, so only one of each type is shown.

The uncorrected PPL errors are again decreased by a factor of about 3 by both the $\triangle \mathrm{PPL}+$ and the COT extrapolation, although the smaller STD of $\triangle \mathrm{PPL}+$ and its lower cost make it more preferable. Compared to that, the application of LEMP2 to PPL brings an additional factor of 3 error reduction with an also improved STD. All approaches preserve the positive sign of the PPL relative error. Compared to PPL, the 
uncorrected error of $E^{\text {rest }}$ is again found to be smaller and very close in magnitude to the $\triangle \mathrm{PPL}+$ errors, but with opposite sign. Except for the loosest threshold, $\epsilon=10^{-4}$, all studied approaches (multiplicative $F^{*}$ scaling, LEMP2, and COT) decrease the MSE. However, the considerable STD values of Fig. 1 indicate that neither the MP2 correlation energy nor the COT measure correlate as well with the FNO truncation error of $E^{\text {rest }}$ as with the PPL error.

Turning our attention to the $(\mathrm{T})$ correction, its uncorrected relative FNO error, compared to the total $\operatorname{CCSD}(\mathrm{T})$ correlation energy, is about half of the error of $E^{\text {rest }}$ but still considerable. About a factor of 2 and 3 error reduction is brought by the $(\mathrm{T}+)$ and COT extrapolation schemes, respectively, both preserving the negative error sign. Again, the orbital component scaled $(\mathrm{T}+)$ and the orbital pair component scaled $(\mathrm{T}+)^{\prime}$ variants are indistinguishable being within $0.03 \%, 0.02 \%$, and $0.01 \%$ of each other for $\epsilon=10^{-4}, \epsilon=10^{-4.5}$, and $\epsilon=10^{-5}$, respectively. Except for the overcorrection at the $\epsilon=10^{-4}$ point, the LEMP2 method is again the best performer due to the similarities of the basis set convergence of the two perturbative schemes: MP2 and (T).

Let us now turn our attention to the accuracy of total CCSD and CCSD(T) correlation energies collected in Fig. 2 for the most promising schemes. MAEs and a logarithmic scale are employed to improve the transparency of the figures. As the (T) correction and its FNO error are relatively small, CCSD and CCSD(T) results share a number of similarities with a few important differences.

First, the error reduction brought by the $\triangle \mathrm{MP} 2$ approach is the smallest and, compared to the case of the closed-shell AW set, its performance is worse for the KAW test set including also open-shell species. The more pronounced role of the PPL and the rest of CCSD terms was also noted in Ref. 62 in the context of AO BSIE reduction of open-shell systems. Comparing the two panels of Fig. 2, $\triangle \mathrm{MP} 2$ apparently benefits from the compensation of the uncorrected positive PPL and negative $E^{\text {rest }}$ and $(\mathrm{T})$ errors. The COT extrapolation improves on $\triangle \mathrm{MP} 2$, but it is not the best for the separate MP2, PPL, or (T) terms, and consequently, it is not the best overall performer. Adding the $\triangle \mathrm{PPL}+$ correction to $\triangle \mathrm{MP} 2$ leads to one of the smallest errors, especially for the two looser $\epsilon$ values, but this is partly due to good cancellation of the imperfectly corrected PPL and uncorrected $E^{\text {rest }}$ terms. This is also apparent from two other observations. First, if a COT extrapolated $E^{\text {rest }}$ correction is added to $\Delta \mathrm{PPL}+\left(\right.$ " $\triangle \mathrm{PPL}+\&$ COT-rest" curve in the top panel), the $E^{\text {rest }}$ error decreases, but the overall performance is considerably worse where $E^{\text {rest }}$ is still notable $\left(\epsilon>10^{-5}\right)$. Second, LEMP2 performs best when the $E^{\text {rest }}$ errors become negligible $\left(\epsilon \leq 10^{-5}\right)$ due to its better performance on the dominating PPL source of error. However, LEMP2 does not benefit from cancellation with negative $E^{\text {rest }}$ errors as, in a considerable amount of cases, LEMP2 overcorrects the $E^{\text {rest }}$ term. This explains its worse performance compared to $\triangle \mathrm{PPL}+$ for the more severe truncations $\left(\epsilon>10^{-5}\right)$. Finally, the combination of the theoretically most promising approaches for each term [" $\Delta \mathrm{MP} 2 \&$ LE-PPL \& COT-rest \& LE-(T)" curve in the bottom panel] indeed leads to the overall best performance, at least for correlation energies.

\subsection{Reaction and atomization energies}

The accuracy of the correction schemes on commonly evaluated energy differences, such as reaction or atomization energies, is also an important performance metric. Thus the closed-shell (CS) and open-shell (OS) reaction energies and atomization energies (AE) collected in the KAW compilation are also evaluated. 

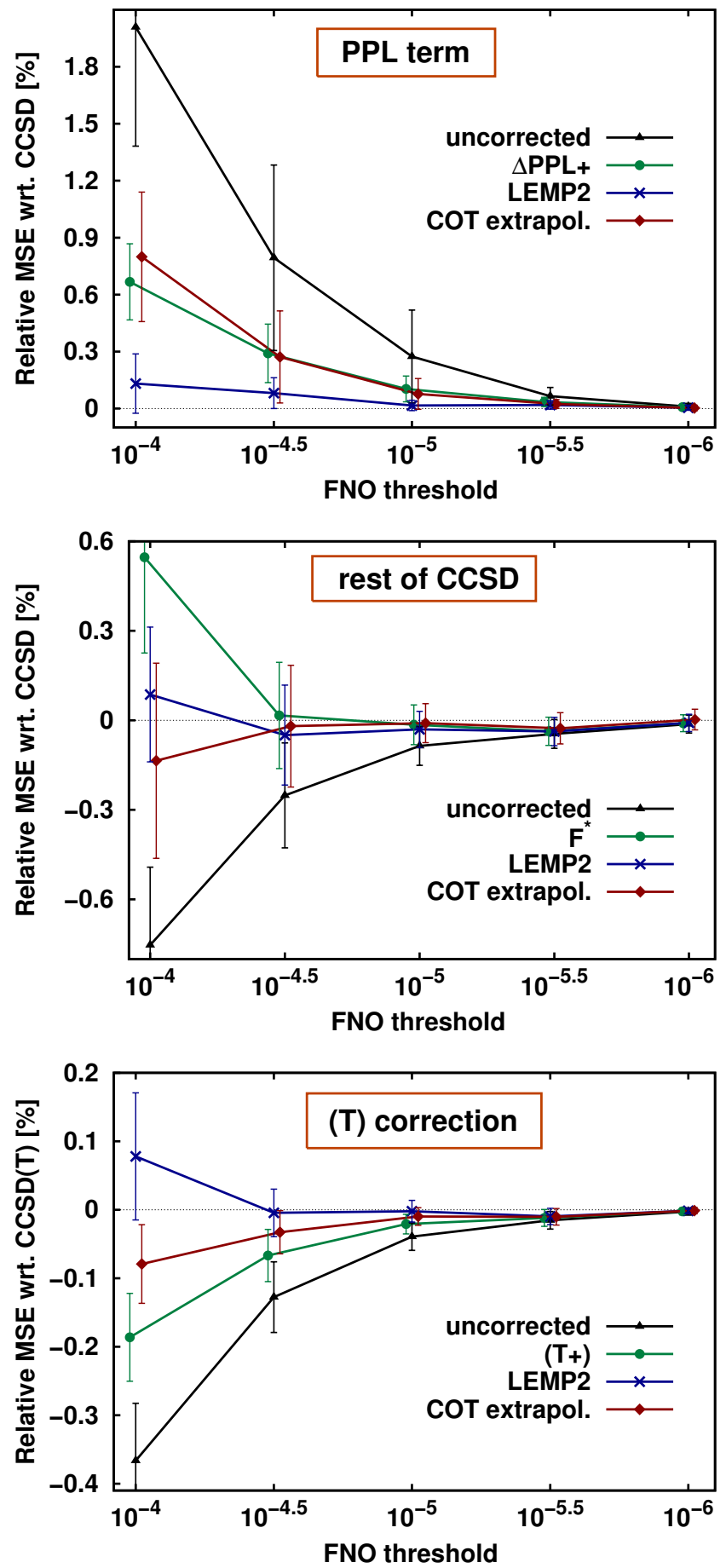

Figure 1. Relative signed error averages for the PPL (top), $E^{\text {rest }}$ (middle), and (T) (bottom) correlation energy contributions (in \%) as a function of the FNO threshold. The error bars represent the STD of the signed relative errors.

Table 2 gathers the separate error components of the MP2, PPL, rest of CCSD, and $(\mathrm{T})$ terms with and without corrections. Here, the reference is the untruncated contribution of the given term to the energy difference, and the MSEs are inspected 

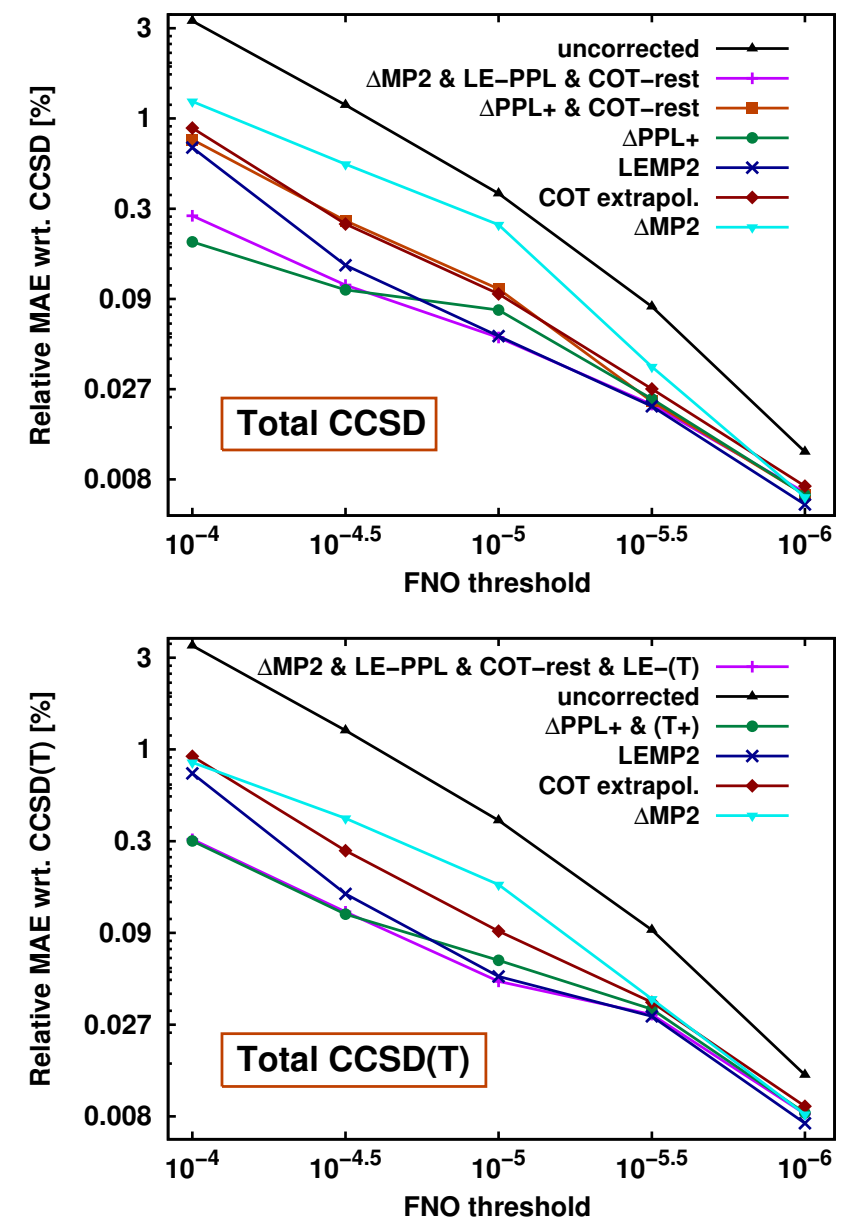

Figure 2. MAEs for CCSD (top) and $\operatorname{CCSD}(\mathrm{T})$ (bottom) correlation energies relative to the respective CCSD and $\operatorname{CCSD}(\mathrm{T})$ references (in \%) as a function of the FNO threshold.

allowing for the discussion of potential error compensations. The MP2 error component, which is perfectly corrected by most schemes, is again the largest but only about 3-4 times larger than the uncorrected PPL and $E^{\text {rest }}$ errors.

The signs of the PPL and $E^{\text {rest }}$ errors remain opposite, similar to the case of correlation energies. The sings of the $E^{\text {rest }}$ and $(\mathrm{T})$ errors also correlate well. This, combined with the observation that the sum of the $E^{\text {rest }}$ and (T) errors is close to the negative of the PPL error explains the good performance of the popular $\triangle \mathrm{MP} 2$ scheme. Interestingly, a large portion of the sizable improvement brought to the PPL correlation contributions by both the $\triangle \mathrm{PPL}+$ and LEMP2 schemes cancels for the CS and OS reaction energies. On the one hand, the AEs and the most loosely truncated $\left(\epsilon=10^{-4}\right)$ reaction energies benefit from both corrections with a noticeably better performance for LEMP2. On the other hand, $\triangle \mathrm{PPL}+$ retains the sign of the PPL error more systematically, hence it is expected to benefit more from cancellation with the $E^{\text {rest }}$ and (T) errors. Considering the PPL term, both $\triangle \mathrm{PPL}+$ and LEMP2 outperforms the COT extrapolation, in accord with the expectation formed on the basis of the PPL correlation contributions.

Markedly different trends are observed for the errors in the rest of CCSD terms. Not surprisingly, the plain $F^{*}$ scaling does more harm than good, while the unsatisfac- 
Table 2. Mean (signed) errors (in $\mathrm{kJ} / \mathrm{mol}$ ) for closed-shell and open-shell reaction energies and atomization energies of the KAW test set as a function of the $\epsilon$ FNO threshold. The MP2, PPL, rest of CCSD, and (T) errors are calculated with respect to the analogous terms of the untruncated reference.

\begin{tabular}{l|ccc|ccc|ccc}
\hline \hline & \multicolumn{3}{|c|}{ Closed-shell reactions } & \multicolumn{3}{c|}{ Open-shell reactions } & \multicolumn{3}{c}{ Atomization energies } \\
FNO threshold, $\epsilon:$ & $10^{-4}$ & $10^{-4.5}$ & $10^{-5}$ & $10^{-4}$ & $10^{-4.5}$ & $10^{-5}$ & $10^{-4}$ & $10^{-4.5}$ & $10^{-5}$ \\
\hline MP2 term & 5.0 & 1.0 & 0.6 & 6.2 & -1.2 & 0.3 & -15.2 & -3.9 \\
PPL term & & & & & & & & -2.6 \\
Uncorrected & -1.7 & -0.3 & -0.2 & -1.9 & 0.6 & -0.1 & 5.0 & 1.3 & 1.1 \\
$\Delta$ PPL+ & -1.6 & -0.6 & -0.3 & -1.2 & -0.1 & -0.2 & 1.6 & 0.4 & 0.4 \\
LEMP2 & 0.3 & -0.5 & -0.2 & 0.7 & -0.3 & -0.1 & -0.7 & -0.03 & 0.1 \\
COT extrap. & -0.9 & 0.5 & 0.02 & -0.4 & 0.8 & 0.1 & 1.5 & -0.02 & 0.2 \\
rest of CCSD & & & & & & & & \\
Uncorrected & 1.4 & 0.4 & 0.1 & 1.2 & -0.5 & -0.1 & -0.6 & 1.0 & 0.3 \\
$F^{*}$ scaled & 1.8 & 1.2 & 0.4 & 1.7 & 0.9 & 0.3 & 1.3 & 1.3 & 1.1 \\
LEMP2 & -1.7 & -0.02 & -0.1 & -2.0 & -0.6 & -0.02 & 3.2 & 1.5 & 0.4 \\
COT extrap. & -0.1 & -0.2 & -0.1 & -0.6 & -1.0 & -0.1 & 1.7 & 1.6 & 0.4 \\
(T) correction & & & & & & & & -0.13 \\
Uncorrected & 0.4 & 0.2 & 0.09 & 0.7 & -0.03 & 0.04 & -1.1 & -0.11 \\
(T+) & 0.4 & 0.1 & 0.03 & 0.3 & -0.06 & 0.02 & -0.05 & 0.17 & 0.01 \\
LEMP2 & 0.2 & -0.2 & -0.03 & 0.3 & -0.01 & 0.01 & 0.14 & 0.24 & 0.04 \\
COT extrap. & 0.5 & -0.1 & -0.01 & 0.5 & -0.21 & -0.02 & -0.32 & 0.22 & 0.02 \\
\hline \hline
\end{tabular}

tory performance of LEMP2 and COT is unexpected considering their corresponding correlation energy errors. Except for the $\epsilon=10^{-4}$ cases, where COT appears to be better, the LEMP2 and COT values are quite close or sometimes are inferior to the uncorrected errors. Considering this point and that the sign of the $E^{\text {rest }}$ error is not retained by these approaches, the best error compensation with $\triangle \mathrm{PPL}+$ is expected in combination with uncorrected $E^{\text {rest }}$. The picture is less clear for LEMP2 as the PPL errors do not appear to correlate well with either the LEMP2 or the COT $E^{\text {rest }}$ errors. For that reason, we further explore both options below.

Finally, regarding the smallest ( $\mathrm{T}$ ) error contributions, the correlation energy improvements provided by the $(\mathrm{T}+)$ and LEMP2 schemes are again largely canceled for most of the reaction and atomization energies. The improvements are significant only with the loosest, $\epsilon=10^{-4}$ threshold in this test set. Considering also the improved correlation energies and the computational cost requirements, the combination of $\triangle \mathrm{PPL}+$ and $(\mathrm{T}+)$ as well as the use of LEMP2 for PPL and $(\mathrm{T})$ appear to be competitive.

Next, let us analyze the accuracy for all terms combined. Since the error contribution of $(\mathrm{T})$ is relatively small, similar conclusions can be drawn for CCSD and CCSD(T), thus only the latter is discussed in detail. Table 3 collects the MAEs of FNO-CCSD(T) compared to the exact reference for the most promising correction schemes. The aim is to bring down the MAE of several $\mathrm{kJ} / \mathrm{mol}$ of the uncorrected FNO-CCSD(T) results within the explored threshold range to an acceptable level of cca. 1-2 kJ/mol. In accord with numerical experience from the literature [36, 58], we also showed that the $\triangle \mathrm{MP} 2$ method is capable of such significant FNO error reduction for a range of different properties [51], such as CS and OS reaction energies of small to medium sized reactants, atomization and ionization processes, or non-covalent interactions. The performance of $\triangle \mathrm{MP} 2$ is also good for the CS and OS reactions of the KAW set (see MAE $=0.2-1.1 \mathrm{~kJ} / \mathrm{mol}$ in Table 3 ), however, the compensation of errors is not as fortunate for the more challenging AEs (MAE=1.4-3.6 kJ/mol).

Compared to FNO-CCSD $(\mathrm{T})+\Delta \mathrm{MP} 2$, the good performance of FNO-CCSD$\mathrm{PPL}(\mathrm{T}+)[$ row " $\triangle \mathrm{PPL}+\&(\mathrm{~T}+)$ "] is more balanced as the AE errors of $0.7-1.6 \mathrm{~kJ} / \mathrm{mol}$ are not significantly worse than those obtained for the CS and OS reaction energies. Except for the AEs, the COT extrapolation of the complete CCSD $(\mathrm{T})$ energy is not 
Table 3. MAEs (in $\mathrm{kJ} / \mathrm{mol}$ ) for $\operatorname{CCSD}(\mathrm{T})$ closed-shell and open-shell reaction energies and atomization energies of the KAW test set as a function of the $\epsilon$ FNO threshold compared to the untruncated reference.

\begin{tabular}{l|ccc|ccc|ccc}
\hline \hline & \multicolumn{3}{|c|}{ Closed-shell reactions } & \multicolumn{3}{c|}{ Open-shell reactions } & \multicolumn{3}{c}{ Atomization energies } \\
FNO threshold, $\epsilon:$ & $10^{-4}$ & $10^{-4.5}$ & $10^{-5}$ & $10^{-4}$ & $10^{-4.5}$ & $10^{-5}$ & $10^{-4}$ & $10^{-4.5}$ & $10^{-5}$ \\
\hline Uncorrected & 7.1 & 2.6 & 1.1 & 7.4 & 2.3 & 0.8 & 12.0 & 3.8 & 1.8 \\
$\Delta \mathrm{MP2}$ & 1.0 & 0.6 & 0.2 & 1.1 & 0.8 & 0.3 & 3.6 & 2.4 & 1.4 \\
$\Delta \mathrm{PPL}+\&(\mathrm{~T}+)$ & 0.9 & 0.5 & 0.3 & 1.3 & 0.7 & 0.3 & 1.4 & 1.6 & 0.7 \\
LEMP2 & 2.6 & 1.3 & 0.4 & 1.8 & 1.2 & 0.2 & 3.2 & 1.8 & 0.6 \\
LEMP2 \& COT-rest & 2.4 & 1.6 & 0.4 & 1.5 & 1.6 & 0.3 & 2.1 & 1.8 & 0.6 \\
COT extrap. & 4.3 & 3.0 & 0.8 & 3.4 & 3.9 & 0.7 & 3.1 & 2.4 & 0.5 \\
\hline \hline
\end{tabular}

consistently better, even compared to the uncorrected results, thus the COT extrapolation is not competitive with the above two methods. The performance of LEMP2 for the complete CCSD(T) energy ("LEMP2" row) as well as of LEMP2 combined with COT extrapolation of only the rest of CCSD terms ("LEMP2 \& COT-rest" row) is also less satisfactory than that of $\triangle \mathrm{MP} 2$ or $\triangle \mathrm{PPL}+\&(\mathrm{~T}+)$. First, the difference between LEMP2 and LEMP2 \& COT-rest is quite small, except for the AEs with $\epsilon=10^{-4}$, due to the smaller size and good compensation of the $E^{\text {rest }}$ errors shown above. Second, while the two LE variants match the accuracy of $\triangle \mathrm{PPL}+\&(\mathrm{~T}+)$ for $\epsilon=10^{-5}$, they are at least $50 \%$ worse for the two looser, $\epsilon<10^{-5}$ values, where $\Delta \mathrm{PPL}+$ can benefit more from error cancellation with the non-negligible $E^{\text {rest }}$ error.

To avoid potential drawbacks characteristic to the MAE measure, in Table 4, we also look at other statistical measures, such as STD and MAX, for the three best methods $[\triangle \mathrm{MP} 2, " \Delta \mathrm{PPL}+\&(\mathrm{~T}+)$ " labeled by $\triangle \mathrm{PPL}$, and "LEMP2 \& COT-rest" labeled by LEMP2']. Table 4 is arranged somewhat differently allowing for a more transparent comparison of the methods across the three different subsets (CS, OS, and AE) for a given FNO threshold value.

Table 4. Statistical measures (MAE, STD, and MAX) for the CCSD(T) errors (in $\mathrm{kJ} / \mathrm{mol}$ ) of closed-shell (CS), and open-shell (OS) reaction energies, and atomization energies (AE) of the KAW test set compared to the untruncated reference. The shorthand labels $\triangle \mathrm{PPL}$ and LEMP2' refer to the above " $\triangle \mathrm{PPL}+\&(\mathrm{~T}+)$ " and "LEMP2 with COT extrapolation for the rest of CCSD terms", respectively.

\begin{tabular}{|c|c|c|c|c|c|c|c|c|c|c|}
\hline \multirow{2}{*}{\multicolumn{2}{|c|}{$\begin{array}{l}\text { FNO } \epsilon: \\
\text { Method: }\end{array}$}} & \multicolumn{3}{|c|}{$10^{-4}$} & \multicolumn{3}{|c|}{$10^{-4.5}$} & \multicolumn{3}{|c|}{$\overline{10}-5$} \\
\hline & & $\Delta \mathrm{MP} 2$ & $\triangle \mathrm{PPL}$ & LEMP2' & $\Delta \mathrm{MP} 2$ & $\triangle \mathrm{PPL}$ & LEMP2' & $\triangle \mathrm{MP} 2$ & $\triangle \mathrm{PPL}$ & LEMP2' \\
\hline \multirow{3}{*}{ MAE } & $\mathrm{CS}$ & 1.0 & 0.9 & 2.4 & 0.6 & 0.5 & 1.6 & 0.2 & 0.3 & 0.4 \\
\hline & OS & 1.1 & 1.3 & 1.5 & 0.8 & 0.7 & 1.6 & 0.2 & 0.3 & 0.3 \\
\hline & $\mathrm{AE}$ & 3.6 & 1.4 & 2.1 & 2.4 & 1.6 & 1.8 & 1.4 & 0.7 & 0.6 \\
\hline \multirow{3}{*}{ STD } & $\mathrm{CS}$ & 1.4 & 1.3 & 4.6 & 0.7 & 0.6 & 2.1 & 0.3 & 0.3 & 0.4 \\
\hline & OS & 1.4 & 2.0 & 2.3 & 1.1 & 0.8 & 1.9 & 0.3 & 0.3 & 0.3 \\
\hline & $\mathrm{AE}$ & 3.2 & 1.6 & 2.3 & 2.3 & 1.4 & 1.6 & 1.3 & 0.6 & 0.4 \\
\hline \multirow{3}{*}{ MAX } & $\mathrm{CS}$ & 4.1 & 4.4 & 19.2 & 1.6 & 1.5 & 5.9 & 0.8 & 0.9 & 1.3 \\
\hline & OS & 3.1 & 9.3 & 7.8 & 2.6 & 3.6 & 7.3 & 0.9 & 1.3 & 1.1 \\
\hline & $\mathrm{AE}$ & 11.7 & 5.4 & 6.9 & 8.9 & 5.6 & 5.7 & 5.3 & 2.6 & 1.7 \\
\hline
\end{tabular}

Comparing the $\triangle \mathrm{MP} 2$ and $\triangle \mathrm{PPL}$ methods first, the close similarity observed for the MAEs of CS and OS reaction energies remain for the STD and MAX values as well. One exception is the $9.3 \mathrm{~kJ} / \mathrm{mol}$ MAX $\triangle \mathrm{PPL}$ error for the OS reaction energy of " $4 \mathrm{NH}_{3}+5 \mathrm{O}_{2} \rightarrow 4 \mathrm{NO}+6 \mathrm{H}_{2} \mathrm{O}$ ", which is a clear outlier because of the large error amplification effect of its sizable stoichiometric coefficients. Indeed, the $\triangle \mathrm{PPL}$ correlation energies are better than with $\triangle \mathrm{MP} 2$ for these four molecules (the respective MAEs are $0.3 \%$ and $0.7 \%$ ), but the corresponding $\Delta \mathrm{MP} 2$ reaction energy of $2.8 \mathrm{~kJ} / \mathrm{mol}$ benefits from better compensation of the reactant and product errors. On the other hand, the AEs obtained with $\triangle \mathrm{PPL}$ are consistently better, by about a factor of 2 , in most cases for all three error measures.

Regarding the LEMP2' results with $\epsilon=10^{-5}$ it is, though by only a small margin, 
the most consistent approach across all subsets of KAW, especially its $1.7 \mathrm{~kJ} / \mathrm{mol}$ MAX error is promising for the most complicated AEs. As the relative importance of the $E^{\text {rest }}$ error grows for larger FNO truncations, the overall performance of LEMP2' deteriorates, especially compared to $\triangle \mathrm{PPL}$. The 5.9 and $19 \mathrm{~kJ} / \mathrm{mol}$ MAX errors obtained for the CS subset with $\epsilon=10^{-4.5}$ and $\epsilon=10^{-4}$, respectively, are particularly high. Upon closer inspection, these MAX errors correspond to the " $2 \mathrm{NH}_{3}+3 \mathrm{~F}_{2} \rightarrow$ $\mathrm{N}_{2}+6 \mathrm{HF}$ " reaction, which is also the most problematic for $\triangle \mathrm{MP} 2$ and $\triangle \mathrm{PPL}$, again because of the large stoichiometric coefficients. Considering the MAE of the relative correlation energy errors of the four participating molecules, LEMP2' is clearly better than $\triangle \mathrm{MP} 2$ and $\triangle \mathrm{PPL}(0.3 \%$ vs $0.6 \%$ and $0.4 \%)$. However, the sign of the error for the HF molecule differs from that of the other three species only for LEMP2', which is amplified by a stoichiometric coefficient of 6 . It is also worthy noting that the linear extrapolation assigned to the $\epsilon=10^{-4}$ point requires the use of the quite crude $\epsilon=10^{-3.5}$ values as well, for which even the $\Delta \mathrm{PPL}$ corrected error of this reaction is $26 \mathrm{~kJ} / \mathrm{mol}$. Thus, in its present form, LEMP2' and also LEMP2 are most reliable for relatively safe FNO truncations. However, they are beneficial for the most challenging cases requiring accurate correlation energies, such as atomization energies.

\subsection{Non-covalent interactions}

The most promising methods are also assessed on non-covalent interactions as these quantities often converge slowly with the employed basis set size and can also pose challenges to second-order perturbation theory [93].

Looking at the FNO-CCSD (T) correlation energies of the species in the A24 compilation [92] (Table 5), the error trends for the uncorrected and various corrected results are very close to those observed for the AW and KAW sets above. It is important to note that the relative correlation energy errors of the monomers and dimers are almost identical in accord with the size-extensive property of the employed corrections.

Table 5. Mean (signed) relative correlation energy errors [in \%] compared to the untruncated CCSD(T) total correlation energy as a function of the $\epsilon$ FNO threshold for the A24 test set.

\begin{tabular}{l|rrr|rrr}
\hline \hline & \multicolumn{3}{|c|}{ Dimer [\%] } & \multicolumn{3}{c}{ Monomer [\%] } \\
FNO threshold, $\epsilon:$ & $10^{-4}$ & $10^{-4.5}$ & $10^{-5}$ & $10^{-4}$ & $10^{-4.5}$ & $10^{-5}$ \\
\hline uncorrected & -4.50 & -1.81 & -0.61 & -4.43 & -1.82 & -0.59 \\
$\Delta$ MP2 & 1.29 & 0.76 & 0.35 & 1.31 & 0.79 & 0.34 \\
$\Delta$ PPL+ & 0.01 & 0.18 & 0.12 & 0.06 & 0.19 & 0.12 \\
LEMP2' & & 0.49 & 0.15 & & 0.51 & 0.15 \\
\hline \hline
\end{tabular}

The corresponding A24 interaction energy errors are collected in Table 6. Here, we find small deviations compared, e.g., to the closed-shell reaction energies of Table 4. While the convergence of both the $\triangle \mathrm{PPL}$ and LEMP2' interaction energy errors remain monotonic with decreasing truncation thresholds, the $\triangle \mathrm{MP} 2$ error measures with $\epsilon=10^{-4}$ are found smaller than the corresponding $\epsilon=10^{-4.5}$ values and appear comparable to the tightest, $\epsilon=10^{-5}$ results. This non-monotonic behavior also deviates from the numerical experience of Ref. 58, where DePrince and Sherrill explored the performance of FNO-CCSD(T) for the interaction energies in the S22 test set. One possible explanation is that the aug-cc-pVDZ basis set was employed in Ref. 58, for which the same $\epsilon=10^{-4}$ threshold leads to a much more conservative decrease in the number of FNOs than in the case of the aug-cc-pVQZ basis employed here [51]. Consequently, the role of the additive MP2 correction is expected to be more pronounced in our aug-cc-pVQZ data, which, in combination with the limitation of MP2 for non- 
covalent interactions, could explain the fortunately small $\triangle \mathrm{MP} 2$ errors. Nonetheless, because of their reliable monotonic improvements, LEMP2' and especially $\triangle$ PPL outperform $\triangle \mathrm{MP} 2$ for the two tighter thresholds. Additionally, all three methods decrease the FNO truncation error by a factor of 5-11 for all explored threshold values, while $\triangle \mathrm{PPL}$ reaches sub-chemical $(0.1 \mathrm{kcal} / \mathrm{mol})$ accuracy already with $\epsilon=10^{-4}$.

Table 6. Statistical measures (MAE, STD, and MAX) for the CCSD(T)/aug-cc-pVQZ errors [in kJ/mol] of noncovalent interaction energies of the A24 test set compared to the untruncated reference. The shorthand labels $\triangle \mathrm{PPL}$ and LEMP2' refer, respectively, to the above " $\triangle \mathrm{PPL}+\&(\mathrm{~T}+)$ " and LEMP2 with COT extrapolation for the rest of CCSD terms.

\begin{tabular}{l|rcc|ccc|ccc}
\hline FNO $\epsilon:$ & \multicolumn{3}{|c|}{$10^{-4}$} & \multicolumn{3}{c|}{$10^{-4.5}$} & \multicolumn{3}{c}{$10^{-5}$} \\
Method: & $\Delta$ MP2 & $\Delta$ PPL & LEMP2, & $\Delta$ MP2 & $\Delta$ PPL & LEMP2' & $\Delta$ MP2 & $\Delta$ PPL & LEMP2' \\
\hline MAE & 0.15 & 0.18 & 0.46 & 0.35 & 0.11 & 0.28 & 0.14 & 0.04 & 0.11 \\
STD & 0.21 & 0.19 & 0.63 & 0.38 & 0.09 & 0.37 & 0.15 & 0.05 & 0.15 \\
MAX & 0.45 & 0.42 & 2.39 & 0.75 & 0.32 & 1.33 & 0.41 & 0.14 & 0.45 \\
\hline \hline
\end{tabular}

While the results in Table 6 are assessed against the CCSD(T)/aug-cc-pVQZ reference, the corresponding counterpoise corrected [94] reference energies and error measures are also evaluated. We found that the accuracy of all three correction schemes is almost identical with and without counterpoise correction, thus the counterpoise corrected set of numbers is not shown for brevity. It is also interesting to point out that the $\triangle \mathrm{PPL}$ corrected FNO-CCSD(T) errors, even with the loosest thresholds, closely approach the intrinsic accuracy of (frozen-core) $\operatorname{CCSD}(\mathrm{T})$ itself, at least based on the core electron correlation and $\operatorname{CCSDT}(\mathrm{Q})$ corrections reported in Ref. 92.

\subsection{Computational requirements}

In our previous report, we explored the computational requirements of the additive MP2 corrected FNO-CCSD(T) method in detail [51] and we recommend the interested reader to consult that extensive analysis. We note one difference here compared to Ref. 51, namely that the NAF approximation [60] was also turned on in Ref. 51 in order to compress the auxiliary basis space, which is unnecessarily large for the density fitting of integrals in the FNO basis. The benefit of this NAF approximation is substantial for our integral-direct $\operatorname{CCSD}(\mathrm{T})$ algorithm since the four-center integral storage can only be avoided via repeated integral assembly, scaling linearly with the number of auxiliary functions. We recommend to take advantage of the highly accurate NAF approximation in the FNO-CCSD $(\mathrm{T})$ context, and it is the default setting in our implementation in the MRCC suite $[84,85]$, but the NAF approach was not employed here in order to explore the pure FNO errors.

As the additive MP2 correction does not require any additional time or storage resource compared to FNO-CCSD $(\mathrm{T})$, the present brief analysis focuses on the speedups obtainable with the $\triangle \mathrm{PPL}$ and LEMP2 corrections. The operation count based theoretical and the corresponding measured speedup values are collected in Table 7 for the cyclohexane entry of the AW collection.

Table 7. Operation count based theoretical and measured speedup values as a function of the retained FNO ratio for cyclohexane in the cc-pVQZ basis set.

\begin{tabular}{lcccccc}
\hline \hline FNO $\epsilon$ & $\%$ FNO & $\left(n_{\mathrm{MO}} / n_{\mathrm{FNO}}\right)^{4}$ & theoretical & $\Delta \mathrm{MP} 2$ & $\Delta \mathrm{PPL}$ & LEMP2 \\
\hline $10^{-5}$ & 63.2 & 6.3 & 6.1 & 5.7 & 5.7 & 4.4 \\
$10^{-4.5}$ & 44.9 & 24.6 & 23.3 & 20.1 & 19.7 & 15.6 \\
$10^{-4}$ & 29.7 & 128.0 & 114.9 & 77.4 & 75.4 & 60.8 \\
\hline \hline
\end{tabular}

The column labeled "theoretical" in Table 7 collects the operation count ratio of 
the rate determining steps with the FNO and the complete virtual basis set taking into account all sixth- and seventh-power scaling, as well as the fifth-power scaling integral assembly operations. This theoretical speedup limit is more informative than

the naive $\left(\frac{n_{\mathrm{MO}}}{n_{\mathrm{FNO}}}\right)^{4}$ speedup estimate, but it is not reached in practice due to the different algorithmic efficiency of the many terms of $\operatorname{CCSD}(\mathrm{T})$ as well as due to the lower-scaling cost reduction of the less operation intensive terms.

As expected, the cost of the $\triangle \mathrm{PPL}$ correction is measured to be about $1-2 \%$ of the total FNO-CCSD(T) computation, thus this little overhead compared to the cost of the $\triangle \mathrm{MP} 2$ approach is amply compensated by the reliability of the $\triangle \mathrm{PPL}$ corrected results. The case of LEMP2 is less clear, although it also provides improved energetics, the extrapolation requires two separate $\mathrm{FNO}-\mathrm{CCSD}(\mathrm{T})$ calculations. With the $10^{0.5}$ threshold ratio employed here, the cost of the second FNO-CCSD(T) run leads to an overhead of about $20 \%$. Nonetheless, compared to the speedups obtained with $\epsilon=10^{-5}$ recommended in our recent paper [51] in combination with $\triangle \mathrm{MP} 2$ corrections, the additional 3-4 and 15 times cost reduction with $10^{-4.5}$ and $10^{-4}$ FNO thresholds, respectively, represent a strong motivation for the application of the more reliable correction schemes.

Finally, the FNO space truncation brings a quadratic- or, in combination with the NAF scheme, cubic-scaling decrease in the memory requirement of our integral-direct $\operatorname{CCSD}(\mathrm{T})$ algorithm, which could also be highly advantageous at the practical limit (1500-2100 orbitals) of our current implementation [51].

\section{Conclusions and outlook}

The reformulation of various FNO corrections in a unified form of scaled MP2-based corrections combined with various energy decomposition ideas led us to a number of novel schemes aiming at reducing the basis set truncation error of FNO-CCSD(T). Similar to the case of the AO basis set incompleteness error (BSIE), the diagrammatic decomposition of Ref. 62 revealed MP2 as the largest, but perfectly correctable FNO error component. The multiplicative PPL correction of Ref. 62 can also reduce the FNO errors of the PPL term by about a factor of 3, leading to an effective cancellation with the error of opposite sign of the remaining, uncorrected CCSD terms in the corresponding $\triangle \mathrm{PPL}^{*}$ correction scheme.

The linear extrapolation (LE) of the FNO-CCSD(T) energies as a function of MP2 energies proposed by us [51] has received an additional theoretical motivation here, and it is found to be a factor of 2-3 more accurate for the correction of both the PPL and (T) correlation energies than any other methods. Utilizing orbital and orbital pair contribution based energy decompositions, we also propose size-consistent variants for the promising $\mathrm{LE}$ and multiplicative schemes, denoted, e.g, as $\triangle \mathrm{PPL}+$ and $(\mathrm{T}+)$. These orbital contribution decomposed variants are also readily applicable in the context of all presently employed local correlation methods [12-14].

Regarding the rest of the CCSD terms, their FNO error is not improved comparably to the improvement in the PPL term with any of the techniques explored here. Thus, the current LE based variants cannot benefit from the compensation of PPL, rest of CCSD, and $(\mathrm{T})$ errors, unlike the popular $\triangle \mathrm{MP} 2$ and the recent $\triangle \mathrm{PPL}$ approaches. Consequently, with somewhat looser FNO thresholds, $\triangle \mathrm{MP} 2$ and $\triangle \mathrm{PPL}+$ perform better for the studied closed- and open-shell reaction energies, while $\Delta \mathrm{PPL}+$ and $(\mathrm{T}+)$ corrected as well as the LE based atomization energies are considerably better 
than the $\triangle \mathrm{MP} 2$ corrected ones. The benefits of the superior LE correlation energies are most apparent with relatively tight FNO thresholds, where the diminishing FNO errors of the rest of CCSD terms cannot cancel the remaining PPL errors.

The error compensation with the $\triangle \mathrm{MP} 2$ and $\triangle \mathrm{PPL}$ approaches is, however, less systematic for more challenging processes involving open-shell species. Thus future studies should focus on the correction of the remaining CCSD terms in order to fully unlock the potential of the LE schemes offered for PPL and (T). One step toward this direction is offered by spin-component decomposition within CCSD. We found that all same-spin terms (MP2, PPL, and rest of CCSD) converge much faster with the FNO threshold than their opposite-spin counterparts, and thus only the FNO error of the opposite-spin component of the rest of CCSD terms remains unsatisfactory.

For now, the $\triangle \mathrm{PPL}+,(\mathrm{T}+)$, and also the LE approaches could contribute to reliable FNO-CCSD $(\mathrm{T})$ results for chemically interesting systems. The implementation of all new schemes is relatively straightforward into existing FNO-CCSD(T) codes. Moreover, the computational overhead of $(\mathrm{T}+)$ is negligible, and the additional cost associated with $\triangle \mathrm{PPL}+$ or LE is about $1-2 \%$ and $20-30 \%$, respectively, of the entire FNO-CCSD $(\mathrm{T})$ computation. In combination, e.g., with the effective and wellparallelized DF-CCSD(T) implementation of the MRCC suite [10], tightly converged $\triangle \mathrm{PPL}+,(\mathrm{T}+)$, or LE corrected FNO-CCSD $(\mathrm{T})$ calculations are feasible up to about 50 atoms even with quadruple- $\zeta$ basis sets [51].

Because of the similarities of the FNO truncation and AO BSIE, the proposed approaches can also be employed in the AO BSIE context. Indeed, the $(\mathrm{T}+)$ method is found to be rather promising [64], and the size-consistent $\triangle \mathrm{PPL}+$ correction can also be useful considering the performance of the original $\triangle \mathrm{PPL}$ approach $[61,62]$. However, the size-consistent implementation of the LE scheme appears to be somewhat more involved in the AO BSIE context. Additional avenues of future studies include the extension of both the FNO truncation and AO BSIE correction schemes to similar many-body methods. Most notably, diagrammatic decomposition based corrections of iterative higher-order CC methods [95] and multiplicative corrections of higher-order perturbative CC approaches, such as the (Q) contribution [96, 97], are envisaged.

\section{Acknowledgments}

This paper is dedicated to Professor John F. Stanton on the happy occasion of his 60th birthday. MK wishes to express his gratitude to John for the fruitful scientific collaborations and continuous support.

\section{Funding}

The authors are grateful for the financial support from the National Research, Development, and Innovation Office (NKFIH, Grant No. KKP126451). The research reported in this paper and carried out at BME has been supported by the NRDI Fund (TKP2020 IES, Grant No. BME-IE-BIO) based on the charter of bolster issued by the NRDI Office under the auspices of the Ministry for Innovation and Technology. The work of PRN is supported by the ÚNKP-20-5 New National Excellence Program of the Ministry for Innovation and Technology from the source of the National Research, Development and Innovation Fund and the János Bolyai Research Scholarship of the Hungarian Academy of Sciences. The computing time granted on the Hungarian HPC 
Infrastructure at NIIF Institute, Hungary, and DECI resources Kay with support from the PRACE aisbl are gratefully acknowledged.

\section{References}

[1] J. Čížek, J. Chem. Phys. 45, 4256 (1966).

[2] J. Gauss, in Encyclopedia of Computational Chemistry, edited by P. R. Schleyer, W. L. Jorgensen, H. F. Schaefer III, P. R. Schreiner and W. Thiel (Wiley, New York, 1998, p. $615)$.

[3] R.J. Bartlett and M. Musial, Rev. Mod. Phys. 79, 291 (2007).

[4] G.D. Purvis III and R.J. Bartlett, J. Chem. Phys. 76, 1910 (1982).

[5] K. Raghavachari, G.W. Trucks, J.A. Pople and M. Head-Gordon, Chem. Phys. Lett. 157, 479 (1989).

[6] A.E. DePrince and C.D. Sherrill, J. Chem. Theory Comput. 9, 2687 (2013).

[7] T. Janowski and P. Pulay, J. Chem. Theory Comput. 4, 1585 (2008).

[8] V.M. Anisimov, G.H. Bauer, K. Chadalavada, R.M. Olson, J.W. Glenski, W.T.C. Kramer, E. Aprà and K. Kowalski, J. Chem. Theory Comput. 10, 4307 (2014).

[9] C. Peng, J.A. Calvin and E.F. Valeev, Int. J. Quantum Chem. 119, e25894 (2019).

[10] L. Gyevi-Nagy, M. Kállay and P.R. Nagy, J. Chem. Theory Comput. 16, 366 (2020).

[11] R.M. Parrish, C.D. Sherrill, E.G. Hohenstein, S.I.L. Kokkila and T.J. Martínez, J. Chem. Phys. 140, 181102 (2014).

[12] P.R. Nagy and M. Kállay, J. Chem. Theory Comput. 15, 5275 (2019).

[13] Q. Ma and H.J. Werner, Wiley Interdiscip. Rev.: Comput. Mol. Sci. 8, e1371 (2018).

[14] Y. Guo, C. Riplinger, U. Becker, D.G. Liakos, Y. Minenkov, L. Cavallo and F. Neese, J. Chem. Phys. 148, 011101 (2018).

[15] G. Schmitz, C. Hättig and D.P. Tew, Phys. Chem. Chem. Phys. 16, 22167 (2014).

[16] W. Li, P. Piecuch, J.R. Gour and S. Li, J. Chem. Phys. 131, 114109 (2009).

[17] T. Helgaker, W. Klopper, H. Koch and J. Noga, J. Chem. Phys. 106, 9639 (1997).

[18] M.S. Marshall, L.A. Burns and C.D. Sherrill, J. Chem. Phys. 135, 194102 (2011).

[19] A. Varandas and P. Piecuch, Chem. Phys. Lett. 430, 448 (2006).

[20] W. Kutzelnigg and W. Klopper, J. Chem. Phys. 94, 1985 (1991).

[21] W. Klopper, F.R. Manby, S.Ten-no and E.F. Valeev, Int. Rev. Phys. Chem. 25, 427 (2006).

[22] C. Hättig, W. Klopper, A. Köhn and D.P. Tew, Chem. Rev. 112, 4 (2012).

[23] L. Adamowicz and R.J. Bartlett, J. Chem. Phys. 86, 6314 (1987).

[24] L. Adamowicz, R.J. Bartlett and J. Sadlej, J. Chem. Phys. 88, 5749 (1988).

[25] C. Sosa, J. Geersten, G.W. Trucks, R.J. Bartlett and J.A. Franz, Chem. Phys. Lett. 159, 148 (1989).

[26] P. Neogrády, M. Pitoňák and M. Urban, Mol. Phys. 103, 2141 (2005).

[27] M. Pitoňák, F. Aquilante, P. Hobza, P. Neogrády, J. Noga and M. Urban, Collect. Czech. Chem. Commun. 76, 713 (2011).

[28] M.C. Clement, J. Zhang, C.A. Lewis, C. Yang and E.F. Valeev, J. Chem. Theory Comput. 14, 4581-4589 (2018).

[29] P.O. Löwdin, Phys. Rev. 97, 1474 (1955).

[30] C. Edmiston and M. Krauss, J. Chem. Phys. 45, 1833 (1966).

[31] T.R. Barr and E.R. Davidson, Phys. Rev. A 1, 644 (1970).

[32] W. Meyer, J. Chem. Phys. 58, 1017 (1973).

[33] P.J. Hay, J. Chem. Phys. 59, 2468 (1973).

[34] R. Ahlrichs, H. Lischka, V. Staemmler and W. Kutzelnigg, J. Chem. Phys. 62, 1225 (1975).

[35] A.G. Taube and R.J. Bartlett, Collect. Czech. Chem. Commun. 70, 837 (2005).

[36] A.G. Taube and R.J. Bartlett, J. Chem. Phys. 128, 164101 (2008).

[37] A. Landau, K. Khistyaev, S. Dolgikh and A.I. Krylov, J. Chem. Phys. 132, 014109 (2010). 
[38] C. Sosa, J. Geertsen, G.W. Trucks, R.J. Bartlett and J.A. Franz, Chem. Phys. Lett. 159, 148 (1989).

[39] Z. Rolik and M. Kállay, J. Chem. Phys. 134, 124111 (2011).

[40] A. Grüneis, G.H. Booth, M. Marsman, J. Spencer, A. Alavi and G. Kresse, J. Chem. Theory Comput. 7, 2780 (2011).

[41] D. Mester, P.R. Nagy and M. Kállay, J. Chem. Phys. 148, 094111 (2018).

[42] B. Ramberger, Z. Sukurma, T. Schäfer and G. Kresse, J. Chem. Phys. 151, 214106 (2019).

[43] J. Segarra-Martí, M. Garavelli and F. Aquilante, J. Chem. Theory Comput. 11, 3772 (2015).

[44] D. Mester, P.R. Nagy and M. Kállay, J. Chem. Phys. 146, 194102 (2017).

[45] P. Pokhilko, D. Izmodenov and A.I. Krylov, J. Chem. Phys. 152, 034105 (2020).

[46] S.D. Folkestad and H. Koch, J. Chem. Theory Comput. 16, 179 (2020).

[47] A. Kumar and T.D. Crawford, J. Phys. Chem. A 121, 708 (2017).

[48] P.R. Nagy, G. Samu and M. Kállay, J. Chem. Theory Comput. 14, 4193 (2018).

[49] M. Pitoňák, F. Holka, P. Neogrády and M. Urban, Theor. Chem. Acc. 768, 79 (2006).

[50] J. Segarra-Martí, M. Garavelli and F. Aquilante, J. Chem. Phys. 148, 034107 (2018).

[51] L. Gyevi-Nagy, M. Kállay and P.R. Nagy, J. Chem. Theory Comput. 17, 860 (2021).

[52] M.R. Nyden and G.A. Petersson, J. Chem. Phys. 75, 1843 (1981).

[53] G.A. Petersson, A. Bennett, T.G. Tensfeldt, M.A. Al-Laham, W.A. Shirley and J. Mantzaris, J. Chem. Phys. 89, 2193 (1988).

[54] G.A. Petersson and M.R. Nyden, J. Chem. Phys. 75, 3423 (1981).

[55] D.S. Ranasinghe and G.A. Petersson, J. Chem. Phys. 138, 144104 (2013).

[56] K.D. Vogiatzis, E.C. Barnes and W. Klopper, Chem. Phys. Lett. 503, 157 (2011).

[57] K.D. Vogiatzis and W. Klopper, Mol. Phys. 111, 2299 (2013).

[58] A.E. DePrince and C.D. Sherrill, J. Chem. Theory Comput. 9, 293 (2013).

[59] K. Sorathia and D.P. Tew, J. Chem. Phys. 153, 174112 (2020).

[60] M. Kállay, J. Chem. Phys. 141, 244113 (2014).

[61] A. Irmler, A. Gallo, F. Hummel and A. Grüneis, Phys. Rev. Lett. 123, 156401 (2019).

[62] A. Irmler and A. Grüneis, J. Chem. Phys. 151, 104107 (2019).

[63] E.F. Valeev, Phys. Chem. Chem. Phys. 10, 106 (2008).

[64] M. Kállay, R.A. Horváth, L. Gyevi-Nagy and P.R. Nagy, J. Chem. Phys. 155, 034107 (2021).

[65] G. Knizia, T.B. Adler and H.J. Werner, J. Chem. Phys. 130, 054104 (2009).

[66] T.D. Crawford and H.F. Schaefer III, Rev. Comp. Chem. 14, 33 (1999).

[67] T. Helgaker, P. Jørgensen and J. Olsen, Molecular Electronic Structure Theory (Wiley, Chichester, 2000).

[68] I. Shavitt and R.J. Bartlett, Many-Body Methods in Chemistry and Physics: MBPT and Coupled-Cluster Theory Cambridge Molecular Science (, , 2009).

[69] P.R. Nagy and M. Kállay, J. Chem. Phys. 146, 214106 (2017).

[70] J.D. Watts, J. Gauss and R.J. Bartlett, J. Chem. Phys. 98, 8718 (1993).

[71] M.J.O. Deegan and P.J. Knowles, Chem. Phys. Lett. 227, 321 (1994).

[72] A. Halkier, T. Helgaker, P. Jørgensen, W. Klopper, H. Koch, J. Olsen and A.K. Wilson, Chem. Phys. Lett. 286, 243 (1998).

[73] T. Takatani, E.G. Hohenstein and C.D. Sherrill, J. Chem. Phys. 128, 124111 (2008).

[74] M. Pitoňák, J. Řezáč and P. Hobza, Phys. Chem. Chem. Phys. 12, 9611 (2010).

[75] S. Grimme, J. Chem. Phys. 118, 9095 (2003).

[76] S. Grimme, L. Goerigk and R.F. Fink, Wiley Interdiscip. Rev.: Comput. Mol. Sci. 2, 886 (2012).

[77] Á. Szabados and P. Nagy, J. Phys. Chem. A 115, 523 (2011).

[78] Z. Rolik and M. Kállay, J. Chem. Phys. 135, 104111 (2011).

[79] Z. Rolik, L. Szegedy, I. Ladjánszki, B. Ladóczki and M. Kállay, J. Chem. Phys. 139, 094105 (2013).

[80] M. Kállay, J. Chem. Phys. 142, 204105 (2015).

[81] P.R. Nagy, G. Samu and M. Kállay, J. Chem. Theory Comput. 12, 4897 (2016). 
[82] P.B. Szabó, J. Csóka, M. Kállay and P.R. Nagy, J. Chem. Theory Comput. 17, 2886 (2021).

[83] K.A. Peterson, M.K. Kesharwani and J.M. Martin, Mol. Phys. 113, 1551 (2015).

[84] M. Kállay, P.R. Nagy, D. Mester, Z. Rolik, G. Samu, J. Csontos, J. Csóka, P.B. Szabó, L. Gyevi-Nagy, B. Hégely, I. Ladjánszki, L. Szegedy, B. Ladóczki, K. Petrov, M. Farkas, P.D. Mezei, Á. Ganyecz and R.A. Horváth, MrCC, a quantum chemical program suite. See https://www.mrcc.hu/ (Accessed June 1, 2021).

[85] M. Kállay, P.R. Nagy, D. Mester, Z. Rolik, G. Samu, J. Csontos, J. Csóka, P.B. Szabó, L. Gyevi-Nagy, B. Hégely, I. Ladjánszki, L. Szegedy, B. Ladóczki, K. Petrov, M. Farkas, P.D. Mezei and Á. Ganyecz, J. Chem. Phys. 152, 074107 (2020).

[86] T.H. Dunning Jr., J. Chem. Phys. 90, 1007 (1989).

[87] R.A. Kendall, T.H. Dunning Jr. and R.J. Harrison, J. Chem. Phys. 96, 6796 (1992).

[88] T.H. Dunning Jr., K.A. Peterson and A.K. Wilson, J. Chem. Phys. 114, 9244 (2001).

[89] F. Weigend, J. Comput. Chem. 29, 167 (2008).

[90] F. Weigend, A. Köhn and C. Hättig, J. Chem. Phys. 116, 3175 (2002).

[91] T.B. Adler and H.J. Werner, J. Chem. Phys. 135, 144117 (2011).

[92] J. Řezáč and P. Hobza, J. Chem. Theory Comput. 9, 2151 (2013).

[93] J. Řezáč and P. Hobza, Chem. Rev. 116, 5038-5071 (2016).

[94] S.F. Boys and F. Bernardi, Mol. Phys. 19, 553 (1970).

[95] M. Kállay and P.R. Surján, J. Chem. Phys. 115, 2945 (2001).

[96] Y.J. Bomble, J.F. Stanton, M. Kállay and J. Gauss, J. Chem. Phys. 123, 054101 (2005).

[97] M. Kállay and J. Gauss, J. Chem. Phys. 123, 214105 (2005). 